\title{
MuSR Beam line design studies
}

\author{
W.W. MacKay \\ Weirich Consulting Services
}

\section{W. Fischer, P. Pile, M. Blaskiewicz Brookhaven National Laboratory}

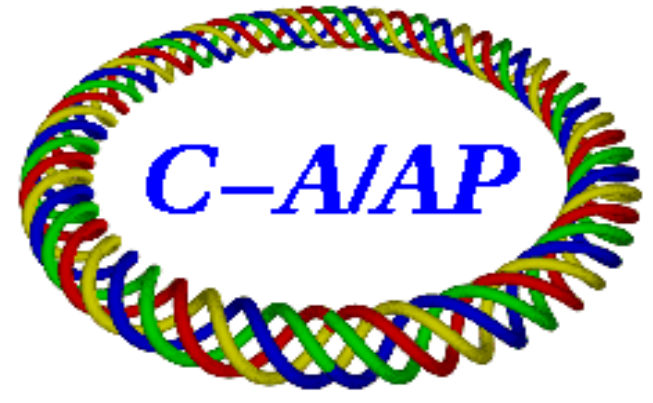

\section{Collider-Accelerator Department Brookhaven National Laboratory Upton, NY 11973}

Notice: This document has been authorized by employees of Brookhaven Science Associates, LLC under Contract No. DE-AC02-98CH10886 with the U.S. Department of Energy. The United States Government retains a nonexclusive, paid-up, irrevocable, world-wide license to publish or reproduce the published form of this document, or allow others to do so, for United States Government purposes. 


\title{
MuSR Beam Line Design Studies
}

\author{
W. W. MacKay* \\ Weirich Consulting Services, Inc., Box 556, Huntersville, NC 28070
}

W. Fischer, P. Pile, and M. Blaskiewicz

BNL, Upton, NY 11973, USA

(Dated: December 13, 2013)

\begin{abstract}
When a substance is implanted with positive muons the precession of their magnetic moments can be used to sample the magnetic properties of the material. The information obtained is complementary to that from NMR, ESR, and neutron scattering. To date, only four user facilities exist in the world but none in the US. We explore the possibility of using the AGS complex at BNL for a $\mu \mathrm{SR}$ facility for the production of positive surface muons. With an incident proton intensity of $10^{14}$ protons per second hitting a $200 \mathrm{~mm}$ long $0.5 \mathrm{~mm}$ thick graphite target, our preliminary design of the beam line could produce low momentum surface muons $(24-30 \mathrm{MeV} / \mathrm{c})$ with a flux of $0.9 \mathrm{MHz} / \mathrm{cm}^{2}$ for experiments.
\end{abstract}

PACS numbers:

Keywords:

\section{INTRODUCTION[1]}

Muon spin rotation, relaxation and resonance $(\mu \mathrm{SR})$ is a powerful technique for studying local magnetic fields in samples. When a positive pion decays at rest into a positive muon, the muon has a kinetic energy of $4.119 \mathrm{MeV}$ (momentum $29.792 \mathrm{MeV} / \mathrm{c}$ ) and its spin is opposite to its direction (negative helicity). If the pion decays near the surface of a target the resulting muons lose little energy, and the result is a beam of muons with a narrow energy distribution and almost $100 \%$ polarization. When these positive muons are implanted in matter with a magnetic field the muons precess at a rate proportional to the local field. When the muon decays the positron momentum is preferentially along the direction of the muon spin. A muon with $4.119 \mathrm{MeV}$ has a typical range of $150 \pm 20 \mathrm{mg} / \mathrm{cm}^{2}[3,13]$ so that such muons are useful to study bulk properties of fairly small samples.

There are four SR user facilities in the world, two pulsed sources at RAL $\left(10^{6} \mu^{+} / \mathrm{s}[8]\right)$ and J-PARC $\left(5 \times 10^{8} \mu^{+} / \mathrm{s}\right.$ $[9])$, and two CW sources at TRIUMF $\left(10^{6} \mu^{+} / \mathrm{s}[8]\right)$, and PSI $\left(4.2 \times 10^{8} \mu^{+} / \mathrm{s}[11]\right)$.

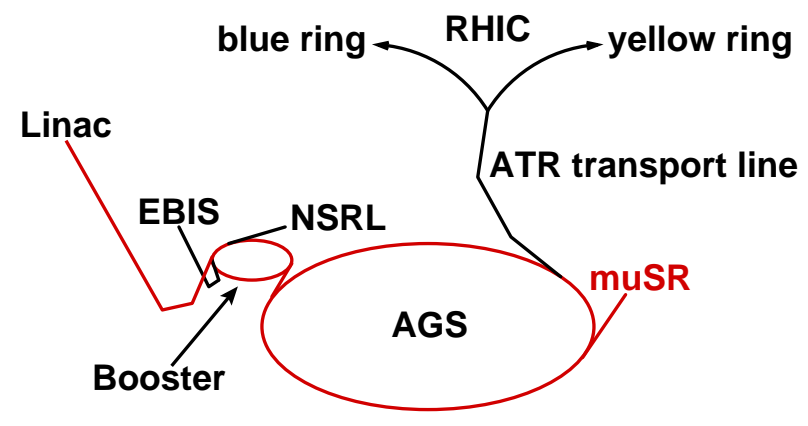

FIG. 1: Schematic of the RHIC-AGS complex to be used for $\mu$ SR (in red).

Material studies using $\mu \mathrm{SR}$ were done at the BNL AGS already in the 1970s and 1980s [5]. We investigate a positive surface muon source at the existing Linac/Booster/AGS complex (Fig. 1), which can be either pulsed with the Booster repetition rate, or DC when the AGS is used as a stretcher ring. In the following we outline possible accelerator, target, and transfer line configurations which have been simulated with the code G4beamline [12]. An early version (Design I) of a beam line (see Fig. 2) was presented[1] at IPAC13 for a $200 \mathrm{~mm}$ long, $50 \mathrm{~mm}$ high, $3 \mathrm{~mm}$ thick graphite target.

*Electronic address: waldo@bnl.gov 
TABLE I: Parameters for surface $\mu^{+}$system

\begin{tabular}{|c|c|c|}
\hline parameter & unit & value \\
\hline \multicolumn{3}{|c|}{ AGS Booster (accelerator for pulsed proton source) } \\
\hline circumference & $\mathrm{m}$ & 201.773 \\
\hline injection energy: $E_{\text {kin }}$ & $\mathrm{MeV}$ & 200 \\
\hline repetition rate & $\mathrm{Hz}$ & 6.67 \\
\hline \multicolumn{3}{|c|}{ AGS (stretcher ring for DC proton source) } \\
\hline circumference & $\mathrm{m}$ & 807.092 \\
\hline injection energy: $E_{\text {kin }}$ & $\mathrm{MeV}$ & 1500 \\
\hline \multicolumn{3}{|c|}{ Parameters for pulsed and DC proton source } \\
\hline extraction energy: $E_{\text {kin }}$ & $\mathrm{MeV}$ & 1500 \\
\hline Emittance: $\pi \epsilon_{95 \%}^{\mathrm{N}}$ & $\mu \mathrm{m}$ & $50 \pi$ \\
\hline proton per pulse/spill & $10^{12}$ & 15 \\
\hline average beam current & $\mu \mathrm{A}$ & 16 \\
\hline average beam power & $\mathrm{kW}$ & 32 \\
\hline \multicolumn{3}{|c|}{ Target for $\mu^{+}$production } \\
\hline material & - & carbon \\
\hline shape & - & rectangular box \\
\hline length $\times$ height $\times$ width & $\mathrm{mm}$ & $200 \times 50 \times 0.5$ \\
\hline cooling & - & radiative \\
\hline$\mu^{+}$flux/side & $\mathrm{GHz}$ & 1.4 \\
\hline \multicolumn{3}{|c|}{ Surface $\mu^{+}$user stations } \\
\hline number of stations & - & 2 \\
\hline modes & - & $6.67 \mathrm{~Hz} / \mathrm{DC}$ \\
\hline \multirow[t]{2}{*}{$\mu^{+}$flux/station } & $\mathrm{MHz}$ & 660 \\
\hline & $\mathrm{MHz} / \mathrm{cm}^{2}$ & 0.91 \\
\hline$\mu^{+} / \mathrm{e}^{+}$ratio & - & 7 \\
\hline
\end{tabular}

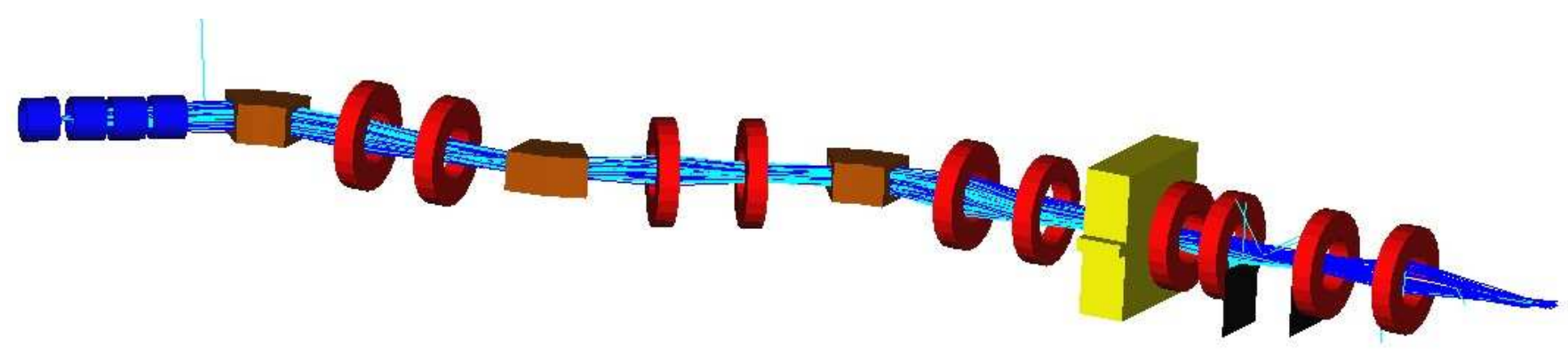

FIG. 2: An early version (Design I) of beam line from Ref. [1] with a length of $21.2 \mathrm{~m}$. Blue cylinders represent solenoids for muon capture; the target is located in the gap just after the first solenoid. The three sector dipoles (brown) bend by respective angles of $-40^{\circ}, 34^{\circ}$, and $-34^{\circ}$. Ten $30 \mathrm{~cm}$ long quadrupoles (red) are shown in doublet pairs along the beam line. A $70 \mathrm{~cm}$ long separator (yellow) with perpendicular electric and magnetic fields deflects positrons and protons from the muon beam. The two vertical scrapers between the eighth and ninth quadrupoles remove deflected positrons. The few protons which make it downstream are removed by the separator, whereas pions will have decayed well upstream of the separator.

More recently, to increase the overall acceptance, we have replaced the first three quadrupole doublets in Fig. 2 with solenoid doublets (see Fig. 3) and shorter drifts around these new solenoids. The second solenoid of each doublet has an equal but opposite field to the first in order to cancel transverse coupling. The main parameters of such a facility are presented in Table I.

\section{SURFACE $\mu^{+}$PRODUCTION}

Surface muons are produced by the decay of stopped pions near the surface of a target. Typically pion beams are produced from a proton beam hitting a target of some material. Positively charged pions decay primarily $(\sim 99.99 \%$ 


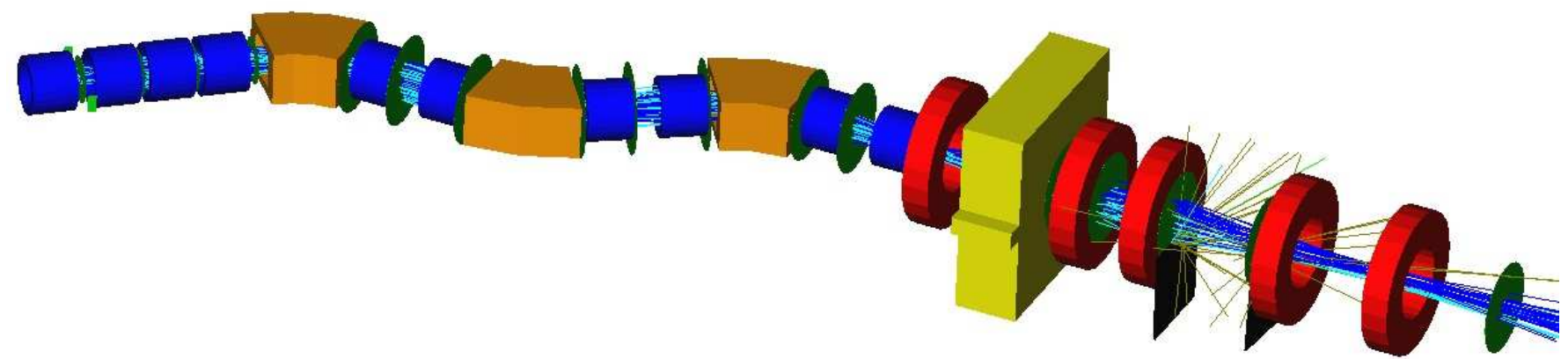

FIG. 3: Modified beam line (Design II) with three solenoidal doublets replacing the quadrupole doublets after each bend. The length of this shortened line is $15.2 \mathrm{~m}$.
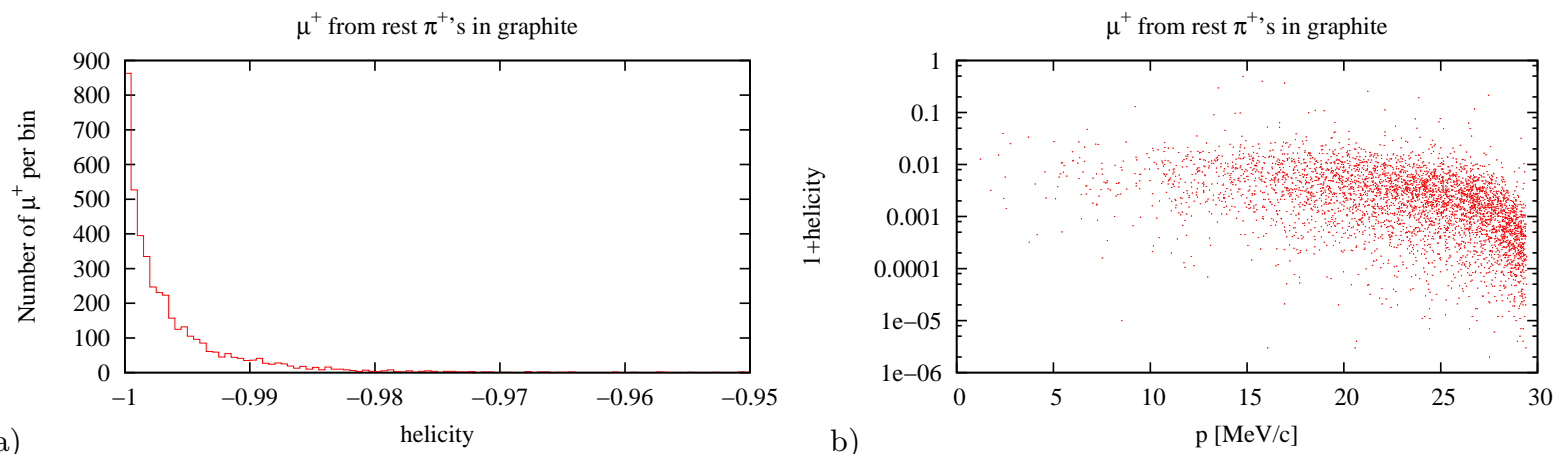

FIG. 4: a) Helicity distribution of surface muons with a bin size of $5 \times 10^{-4}$. b) Deviation of helicity from -1 vs momentum of muons. The small spread in helicity is due to scattering within the target.

of the time) via the two-body reaction

$$
\pi^{+} \rightarrow \mu^{+}+\nu_{\mu}
$$

in the rest frame of the $\pi^{+}$, the resulting $\mu^{+}$momentum is given by

$$
p_{\mu}=\frac{m_{\pi}^{2}-m_{\mu}^{2}}{2 m_{\pi}} c=29.7920 \mathrm{MeV} / \mathrm{c},
$$

i. e. with a kinetic energy of $4.1198 \mathrm{MeV}$.

Since pions have spin zero, the muons are produced with negative helicity $(h=-1)$, i. e. with polarization opposite to the direction of motion, as shown in Fig. 4 for a simulation with a uniform distribution of at-rest $\pi^{+}$within the
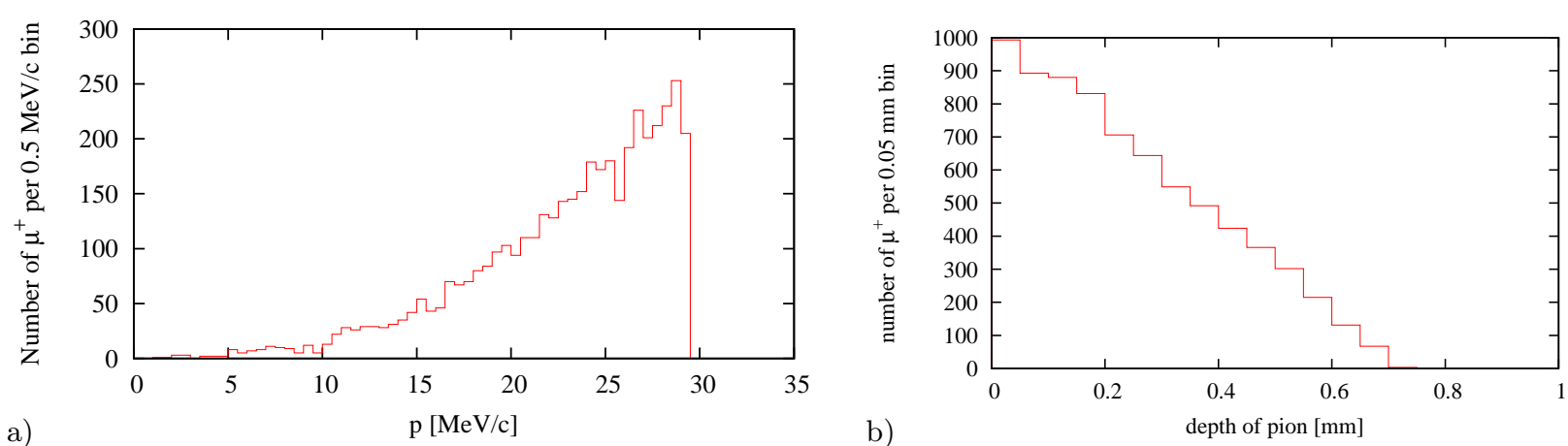

FIG. 5: a) Momentum distribution of surface muons from uniformly distributed at-rest $\pi^{+}$within the target. b) Depth distribution of $\mu^{+}$production within the target. Exiting surface muons come only from pions which stop within the last $0.7 \mathrm{~mm}$ of the graphite target. 

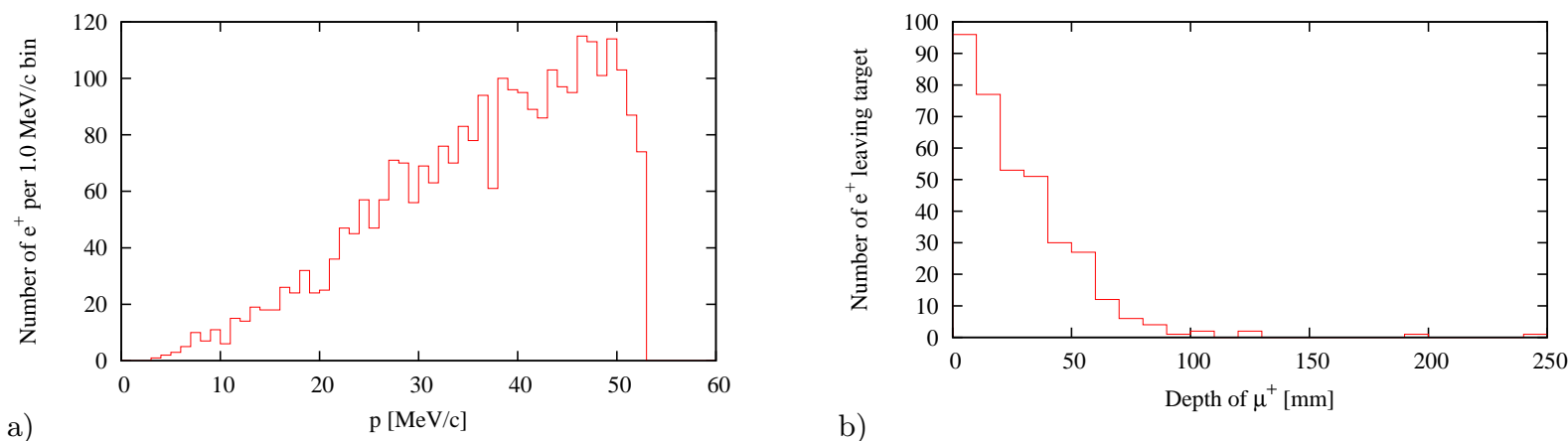

b)

FIG. 6: a) Momentum distribution of $\mathrm{e}^{+}$from at-rest $\mu^{+}$in $50 \mathrm{~cm}$ thick graphite target. b) Creation depths of $\mathrm{e}^{+}$exiting the target from a uniform depth distribution of muons at rest.

target. Even though the muons from stopped pions are produced with the same momentum, the muons will lose energy as they pass through target material depending on their depth and angle of trajectory. This produces a distribution of momenta for the surface muons as shown in Fig. 5a. Due to the energy loss of muons in the production target, only muons close to the surface can escape from the target; for a graphite target, muons come from the last $0.7 \mathrm{~mm}$ (see Fig. 5b).

Positive muons which stop in the target decay into positrons almost always through the decay channel

$$
\mu^{+} \rightarrow \mathrm{e}^{+}+\bar{\nu}_{\mu}+\nu_{\mathrm{e}}
$$

This is a three-body decay, so in the rest-system of the muon, the momentum distribution of the daughter positrons has a distribution as shown in Fig. 6a with a maximum momentum of $52.828 \mathrm{MeV} / \mathrm{c}$ that is higher than for the surface muons. Therefore the positrons from muons at rest can come from deeper within the target. Fig. $6 \mathrm{~b}$ shows the distribution of depths of escaping positrons for from a large graphite target for evenly distributed $\mu^{+}$at rest within the target.

\section{SOLENOIDS AND COUPLING}

Before proceeding with the description of muon capture, let us review a few points about solenoids and coupling. On axis, a solenoid of length $l$ and average radius $a$ has a longitudinal field component given by $[6]$

$$
B_{s}(s)=B_{0} \frac{\sqrt{l^{2}+4 a^{2}}}{2 l} \times\left[\frac{s}{\sqrt{s^{2}+a^{2}}}+\frac{l-s}{\sqrt{(l-s)^{2}+a^{2}}}\right],
$$

where $B_{0}$ is the central field expected in the limit of an infinitely long solenoid. Near the axis, the radial field component is given by

$$
\begin{aligned}
B_{r}(r, s) & =-\int \frac{\partial B_{s}}{\partial s} d r \\
& =-B_{0} \frac{\sqrt{l^{2}+4 a^{2}}}{2 l}\left[\frac{a^{2}}{\left(s^{2}+a^{2}\right)^{3 / 2}}-\frac{a^{2}}{\left((s-l)^{2}+a^{2}\right)^{3 / 2}}\right] r
\end{aligned}
$$

For a long solenoid, the transport $4 \times 4$ matrix may be written as $[2,7]$

$$
\mathbf{M}_{\mathrm{sol}}=\mathbf{R} \mathbf{M}_{\mathrm{f}}=\mathbf{M}_{\mathrm{f}} \mathbf{R}
$$

with a simple rotation matrix

and symmetric focusing lens

$$
\mathbf{R}=\left(\begin{array}{cc}
\mathbf{I} \cos \frac{k l}{2} & \mathbf{I} \sin \frac{k l}{2} \\
-\mathbf{I} \sin \frac{k l}{2} & \mathbf{I} \cos \frac{k l}{2}
\end{array}\right)
$$

$$
\mathbf{M}_{\mathrm{f}}=\left(\begin{array}{cc}
\mathbf{F} & \mathbf{0} \\
\mathbf{0} & \mathbf{F}
\end{array}\right)
$$

where $\mathbf{I}$ is the $2 \times 2$ identity matrix, and 
a)

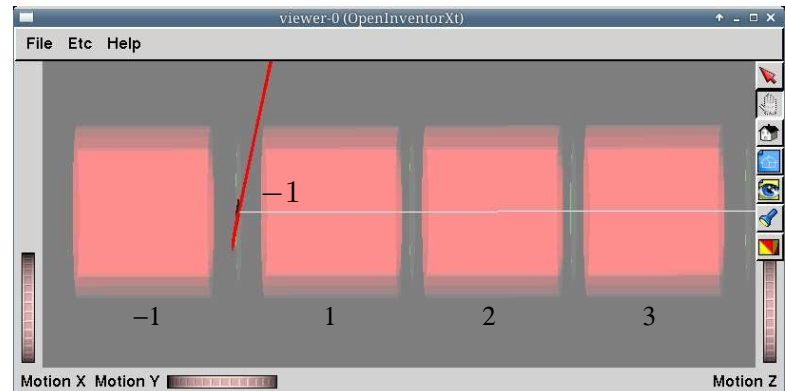

FIG. 7: a) Layout of $\mu$ capture solenoids with 3 solenoids downstream of the target and one for extra field shaping upstream of the target. The solenoids are numbered as shown counting outward from the target with the "- 1 " solenoid upstream of the target. The proton beam (red) enters from the side perpendicular to the solenoid axis. Muons are captured by the three solenoids to the right with the extra solenoid on the left providing extra field shaping. (The thin greenish disks shown between solenoids are virtual detectors used in the G4beamline simulations to count particles, but do not affect the beam.) b) Simulation of $\mu^{+}$(dark blue) capture from pions at rest just $0.1 \mathrm{~mm}$ downstream of the target.

$$
\mathbf{F}=\left(\begin{array}{cc}
\cos \frac{k l}{2} & \frac{2}{k} \sin \frac{k l}{2} \\
-\frac{k}{2} \sin \frac{k l}{2} & \cos \frac{k l}{2}
\end{array}\right) .
$$

Here the constant $k$ is the central field divided by the beam's rigidity:

$$
k=\frac{q B_{0}}{p} .
$$

This decomposition of the solenoid matrix into a rotation and an uncoupled lens with equal focusing in both planes, allows reordering of the matrices for a number of solenoids and drifts where we may combine all the rotation matrices into a single rotation at either end of an uncoupled matrix, e. g.

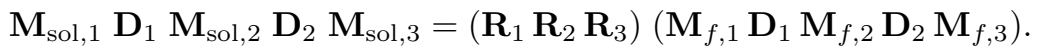

So the overall rotation angle for a group of coaxial solenoids may be written as

$$
\Theta=\frac{q}{2 p} \int B_{s} d s
$$

and the net rotation matrix of the form Eq. 7 will become an identity matrix if $\Theta$ is some multiple of $2 \pi$. More restrictively, if we require that $\Theta=0$, then the solenoids will be decoupled for a particle of any rigidity. Even though Eq. 6 is given for a long solenoid $(l \gg a)$, rotational decomposition is more general so that the near-axis transport matrix of a general cylindrically symmetric magnetic field will be decoupled when $\Theta=0$.

\section{MUON CAPTURE}

Low energy surface muons exit the target with a large divergence, and are captured by a group of nearby largeaperture solenoid lenses as shown in Fig. 7a. The primary proton beam enters from the side perpendicular to the solenoid axis and passes down the length of the target. Fig. 7b shows a simulation of the capture of $\mu^{+}$from a plane of rest $\pi^{+}$just downstream of the target.

In order to see if a longer target which was tilted relative to the solenoid axis might yield a larger effective area for surface muon production, we compared the two target configurations shown in Fig. 8. The target in both cases was a graphite target of dimensions $400 \times 50 \times 6 \mathrm{~mm}$. We saw no appreciable difference in the number of muons below $30 \mathrm{MeV} / \mathrm{c}$ collected in the detector downstream of solenoid 3. Even though the target is long, some muons which leave the target are bent back into the target in the tilted case. For the nontilted case, the captured muons were coming only from the about the middle $100 \mathrm{~mm}$ portion of the target

Using the solenoid settings $B_{\mathrm{sol}, 1}=0.43 \mathrm{~T}, B_{\mathrm{sol}, 2}=-0.2 \mathrm{~T}$, and $B_{\mathrm{sol}, 3}=-0.23 \mathrm{~T}$, we studied $\mu^{+}$capture for various locations of a point source of muons at the target. Initially, we used a point source of muons with rms opening angles having $\sigma_{x^{\prime}}=\sigma_{y^{\prime}}=0.35 \mathrm{mrad}$. Comparing the plots in Fig. 9, we see the increase in angular acceptance with $B_{\mathrm{sol},-1}=0.43 \mathrm{~T}$. The point source acceptance for a source on axis,

$$
A\left(\theta_{r}\right)=2 \pi \int_{0}^{\theta_{r}} \sin \theta d \theta=2 \pi\left(1-\cos \theta_{r}\right),
$$


a)

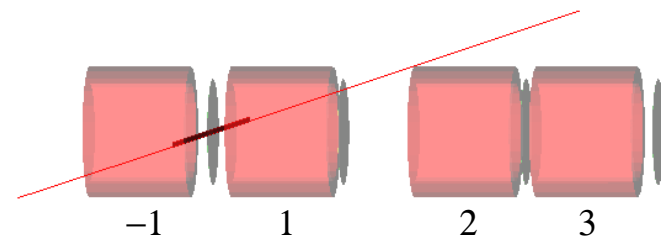

b)

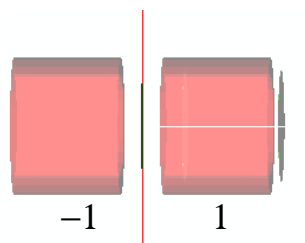

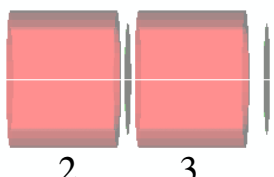

3

FIG. 8: a) A $400 \mathrm{~mm}$ long target angled at $20^{\circ}$ relative to the solenoid axis with a larger gap between solenoids 1 and 2 to allow for the proton beam to exit in the gap. The three solenoid fields ( 1 to 3 ) were $0.62,-0.42,-0.2 \mathrm{~T}$ were tuned for maximum transmission of $\mu^{+}$through the last solenoid. b) Same geometry as a, but with the target and proton beam perpendicular to the solenoid axis. For this orientation the optimized fields were $0.57,-0.43,-0.14 \mathrm{~T}$. In both cases, $B_{\mathrm{sol},-1}=B_{\mathrm{sol}, 1}$.

a)

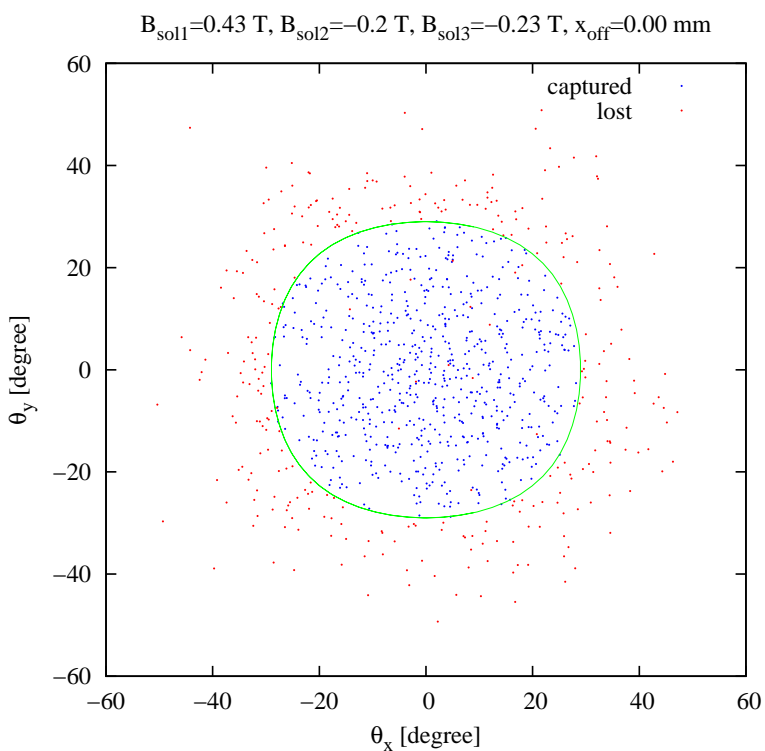

$$
\mathrm{B}_{\text {sol1 }}=0.43 \mathrm{~T}, \mathrm{~B}_{\mathrm{sol} 2}=-0.2 \mathrm{~T}, \mathrm{~B}_{\mathrm{sol} 3}=-0.23 \mathrm{~T}, \mathrm{x}_{\mathrm{off}}=0.00 \mathrm{~mm}
$$

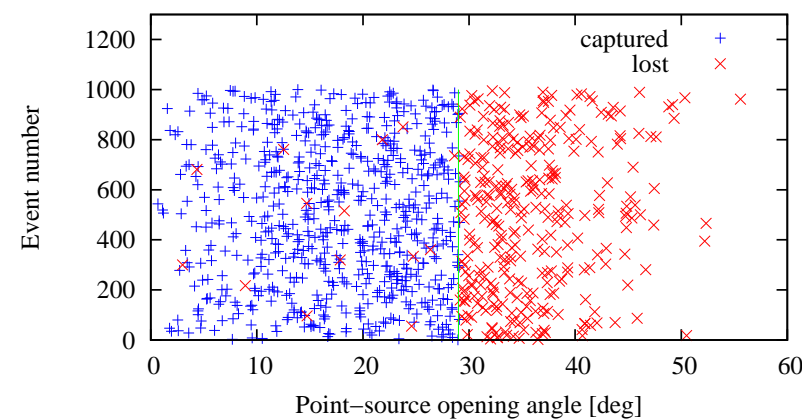

$\mathrm{B}_{\mathrm{sol} 1}=0.43 \mathrm{~T}, \mathrm{~B}_{\mathrm{sol} 2}=-0.2 \mathrm{~T}, \mathrm{~B}_{\mathrm{sol} 3}=-0.23 \mathrm{~T}, \mathrm{x}_{\mathrm{off}}=0.00 \mathrm{~mm}$

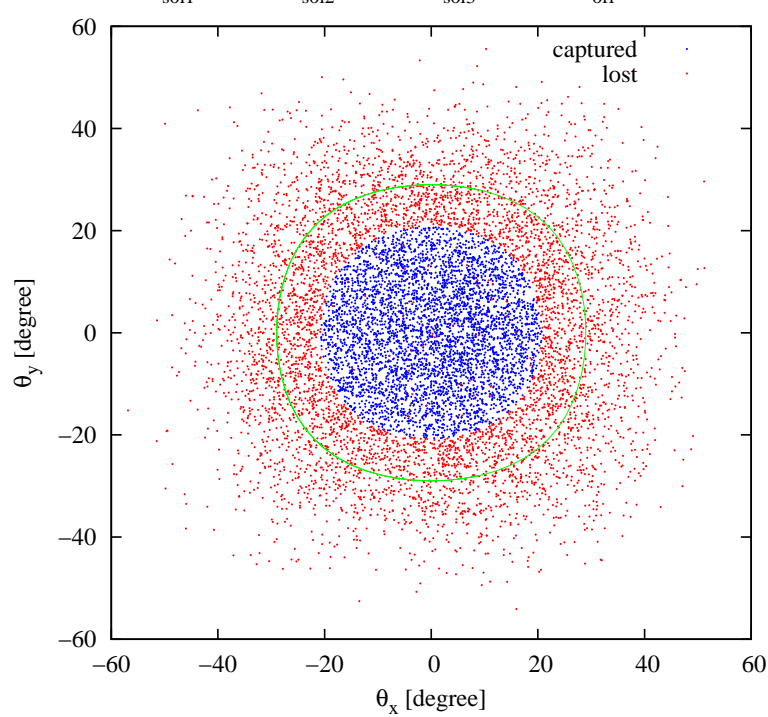

b)

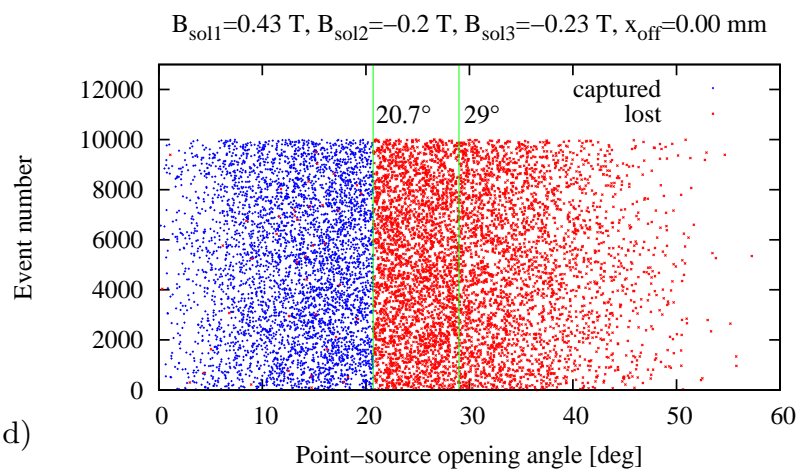

FIG. 9: Capture acceptance of monochromatic $\mu^{+}$from a centered point source at the target. Plotted angles are for a detector at the source with blue (red) indicating muons which hit (missed) the downstream detector after solenoid 3 . The green curves are are for a $\theta_{r}=29^{\circ}$ opening angle. Left: for $B_{\mathrm{sol},-1}=0.43 \mathrm{~T}$. Right: for $B_{\mathrm{sol},-1}=0$. Note that in a and b, the green contour is not circular since in polar coordinates $\theta_{x}=\tan ^{-1}\left[\tan \theta_{r} \cos \phi\right]$ and $\theta_{y}=\tan ^{-1}\left[\tan \theta_{r} \sin \phi\right]$ so $\theta_{r} \leq \sqrt{\theta_{x}^{2}+\theta_{y}^{2}}$.

increases from $A\left(20.7^{\circ}\right)=406 \mathrm{msr}$ to $A\left(29^{\circ}\right)=787 \mathrm{msr}$ because of this solenoid. For comparison, Miyake quoted an acceptance of $400 \mathrm{msr}$ for the normal conducting capture solenoid of the J-PARC MLF MUSE [9, 10].

Fig. 10 shows how the number of captured muons varies for both $B_{\text {sol, }-1}$ and distance of the point source from the solenoid axis. Not much is to be gained for a target longer than $200 \mathrm{~mm}$. The Axial field profile through the solenoids is plotted in Fig. 11a. For a uniform distribution $(200 \times 100 \mathrm{~mm})$ of 10,000 at-rest pions replacing the target, Fig. 11b shows that the capture efficiency increases considerably with $B_{\mathrm{sol},-1}$ up to about $1.6 \mathrm{~T}$.

We then reoptimized the solenoid fields, having constrained $B_{\mathrm{sol},-1}=1.5 \mathrm{~T}$ for maximum transport, while using 


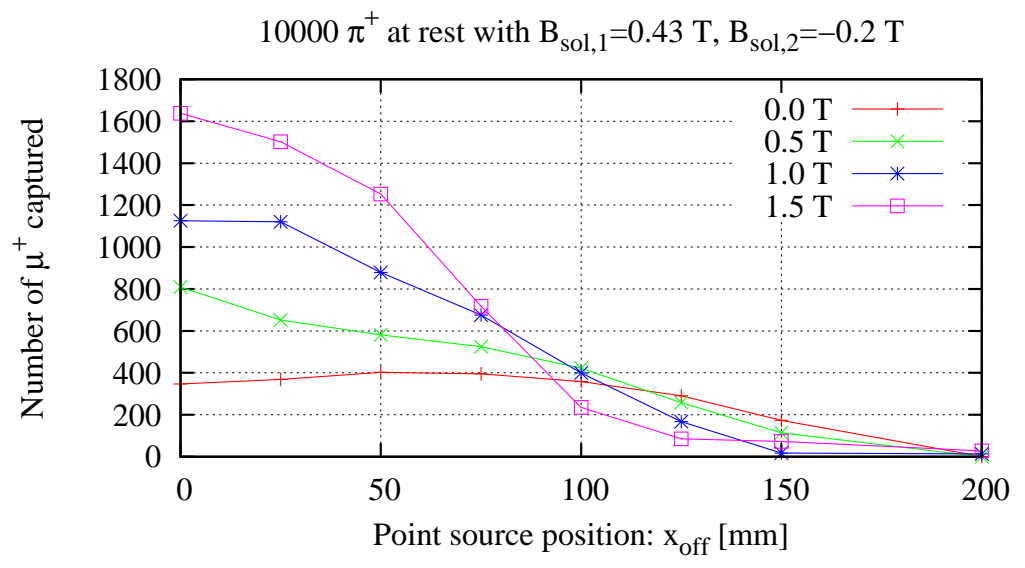

FIG. 10: Muon capture efficiency for several point sources of 10,000 rest pions versus source distance from solenoid axis. $B_{\mathrm{sol},-1}=B_{\mathrm{sol}, 1}=0.43 \mathrm{~T}, B_{\mathrm{sol}, 2}=-0.2 \mathrm{~T}$, and $B_{\mathrm{sol}, 3}=-0.23 \mathrm{~T}$.
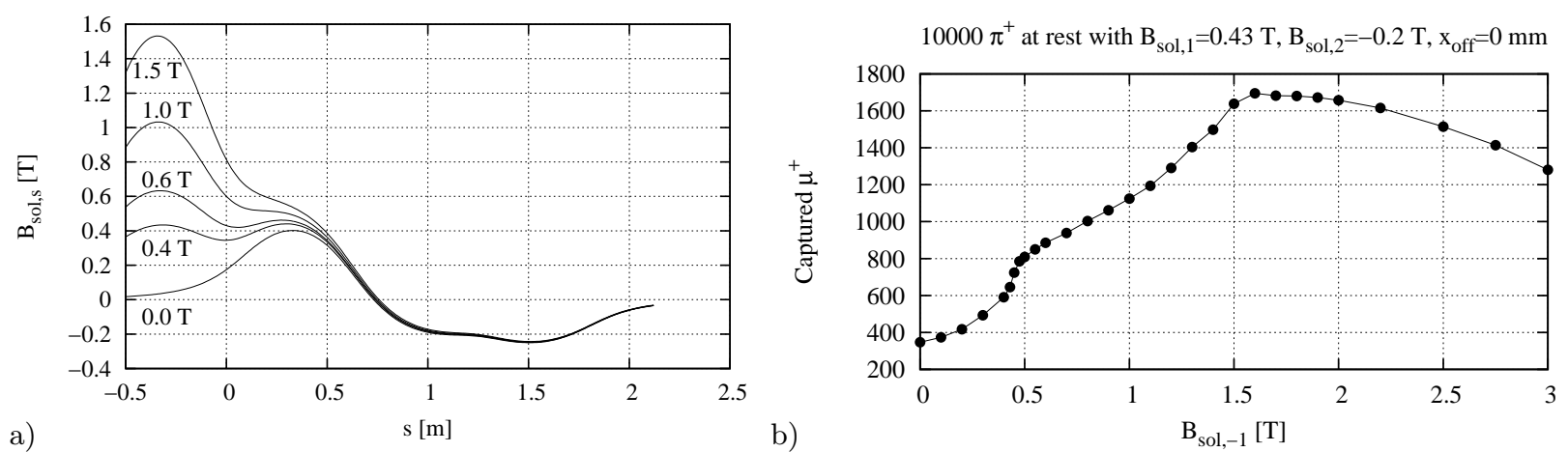

FIG. 11: a) Axial field profile through the solenoids for various settings of $B_{\text {sol, }-1}$. Note that even though the setting of solenoid 1 with center at $s=0.35 \mathrm{~m}$ is $B_{\mathrm{sol}, 2}=0.43 \mathrm{~T}$, its central field is only $0.4 \mathrm{~T}$ in the lowest curve $(-1$ off $)$ since the fringe fields of solenoids 2 and 3 are negative and must be superimposed with solenoid 1. b) Capture efficiency from a flat distribution of 10,000 pions at rest versus $B_{\mathrm{sol},-1}$.

the program syrk4track (see $\S \mathrm{X}$ ) to calculate a transport matrix and decouple the $3 \mathrm{~m}$ long section from the target. For the final fields,

$$
\begin{aligned}
B_{\mathrm{sol},-1} & =1.5 \mathrm{~T} \\
B_{\mathrm{sol}, 1} & =0.59 \mathrm{~T}, \\
B_{\mathrm{sol}, 2} & =-0.4 \mathrm{~T} \\
B_{\mathrm{sol}, 3} & =-0.32 \mathrm{~T},
\end{aligned}
$$

we obtained the field profile shown in Fig. 12 and the $4 \times 4$ sufficiently decoupled transport matrix

$$
\left(\begin{array}{rrrr}
2.007239 & -0.596408 & 0.000003 & -0.000001 \\
3.003413 & -0.394202 & 0.000005 & -0.000001 \\
-0.000003 & 0.000001 & 2.007239 & -0.596408 \\
-0.000005 & 0.000001 & 3.003413 & -0.394202
\end{array}\right) .
$$

Recalling Eq. 12, integration of the field in Fig. 12 gives

$$
\frac{p}{q} \Theta=\int_{0}^{3 \mathrm{~m}} B_{s} d s=3 \times 10^{-4} \mathrm{Tm},
$$




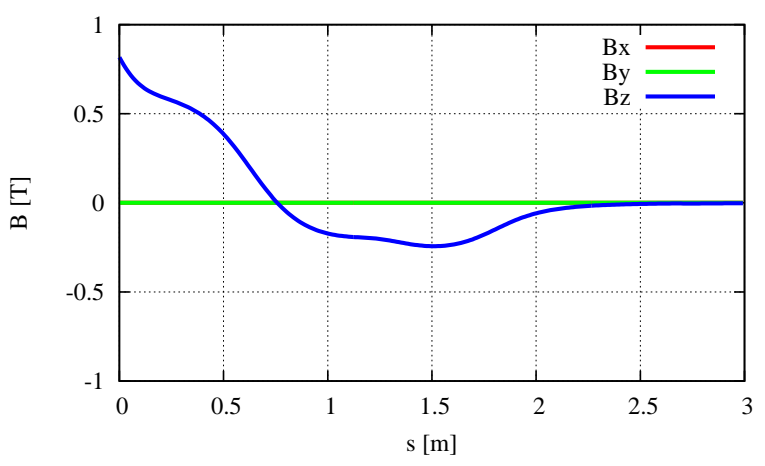

FIG. 12: Modeled magnetic components of the front end solenoids from syrk4track integration.

which for a $29.792 \mathrm{MeV} / \mathrm{c} \mu^{+}$corresponds to a net rotation of $\Theta=3.9 \mathrm{mrad}$. For comparison integrating the field magnitude gives

$$
\int_{0}^{3 \mathrm{~m}}\left|B_{s}\right| d s=0.79 \mathrm{Tm} .
$$

It should be noted that the decoupling may not be quite this simple since the fringe of the end solenoid will overlap with the fringe of the first dipole.

\section{SEPARATOR}

While trajectories of particles with identical charges $q$ and momentum $p_{\text {ref }}$ have the same radii of curvature in a perpendicular magnetic field, their velocities will depend on mass $m$ :

$$
\vec{v}_{\text {ref }}=\frac{p_{\text {ref }} c}{\sqrt{p_{\text {ref }}^{2}+m^{2} c^{2}}} \hat{z},
$$

where we have assumed that the reference particle moves along the $z$-axis. In order to separate particles of different masses, we will use the perpendicular electric and magnetic fields of a Wien filter[15, 16].

Since we have chosen to have horizontal bends, it is better to separate the beams vertically, i. e. in the plane with minimum dispersion. For a reference $\mu^{+}$there should be no deflection from the Lorentz force:

$$
\vec{F}=q\left(\vec{E}+\vec{v}_{\text {ref }, \mu^{+}} \times \vec{B}\right)=0,
$$

so for a given vertical electric field $E_{y}$, the magnetic field should be

$$
B_{x}=-\frac{E_{y}}{v_{\text {ref }, \mu^{+}}},
$$

where we have assumed a hard-edged model of electric and magnetic fields of the same length.

Fig. 13 demonstrates separation of protons and positrons from $\mu^{+}$for a $70 \mathrm{~cm}$ long separator with a vertical gap of $20 \mathrm{~cm}$, with

$$
\begin{aligned}
V_{\text {sep }} & =300 \mathrm{kV}, \\
E_{y} & =1.5 \mathrm{MV} / \mathrm{m}, \\
B_{x} & =18.4 \mathrm{mT} .
\end{aligned}
$$

While the $\mu^{+}$particle with initial $p_{y}=0$ does not change momentum, muons with $p_{y}= \pm 0.1 \mathrm{MeV} / \mathrm{c}$ change momentum by $\pm 13 \mathrm{keV} / \mathrm{c}$ respectively, since $\int E_{y} d y \neq 0$.

\section{DESCRIPTION OF BEAM LINE}

We describe two versions of the beam line: 1) from the IPAC2013 paper[1], and 2) a new design with a shorter beam line and larger acceptance from a longer target and higher field capture solenoids. A third version of the beam line with solenoidal bends was considered briefly and is described in the appendix $\S$ XI. 


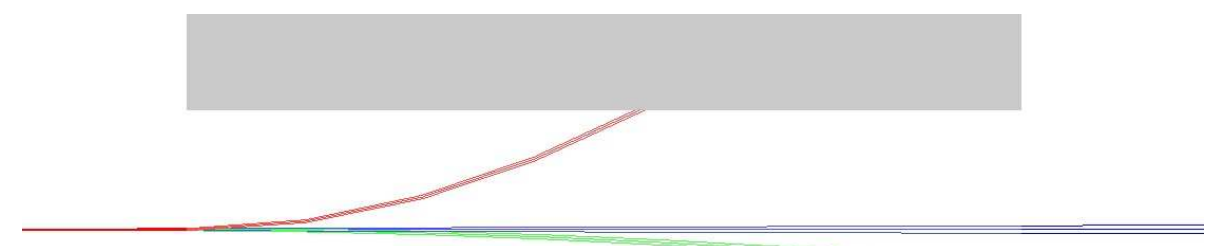

FIG. 13: Wien filter used as separator (with vertical plates shown in gray). Trajectories are shown for protons (red), $\mu^{+}($dark blue), and $\mathrm{e}^{+}$(green) with initial $p=29.792 \mathrm{MeV} / \mathrm{c}$ and $p_{y}=0, \pm 0.1 \mathrm{MeV} / \mathrm{c}$.

TABLE II: Parameters of Design I.

TABLE III: Parameters of Design II

\begin{tabular}{|c|c|c|c|c|c|c|c|c|c|}
\hline Element & $\begin{array}{l}s_{\text {up }} \\
{[\mathrm{m}]}\end{array}$ & $\begin{array}{c}\text { Length } \\
{[\mathrm{m}]}\end{array}$ & $\begin{array}{c}\text { Aperture } \\
{[\mathrm{m}]}\end{array}$ & Strength & Element & $\begin{array}{l}s_{\text {up }} \\
{[\mathrm{m}]}\end{array}$ & $\begin{array}{c}\text { Length } \\
{[\mathrm{m}]}\end{array}$ & $\begin{array}{c}\text { Aperture } \\
{[\mathrm{m}]}\end{array}$ & Strength \\
\hline Solenoids & & & (radius) & {$[\mathrm{T}]$} & Solenoids & & & (radius) & [T] \\
\hline $\mathrm{S}(-1)$ & -0.6 & 0.5 & 0.24 & -0.335 & $\mathrm{~S}(-1)$ & -0.100 & 0.5 & 0.24 & 1.50 \\
\hline $\mathrm{S} 2$ & 0.1 & 0.5 & 0.24 & -0.335 & $\mathrm{~S} 1$ & 0.100 & 0.5 & 0.24 & 0.59 \\
\hline $\mathrm{S} 2$ & 0.7 & 0.5 & 0.24 & 0.280 & $\mathrm{~S} 2$ & 0.700 & 0.5 & 0.24 & -0.40 \\
\hline S3 & 1.3 & 0.5 & 0.24 & 0.055 & S3 & 1.300 & 0.5 & 0.24 & -0.32 \\
\hline Sector bends & & & $(h \times w)$ & {$[\mathrm{T}]$} & SA1 & 3.100 & 0.5 & 0.24 & 0.25 \\
\hline $\mathrm{B} 1\left(-40^{\circ}\right)$ & 2.51 & 1.0 & $0.4 \times 0.5$ & 0.0693 & SA2 & 3.899 & 0.5 & 0.24 & -0.25 \\
\hline $\mathrm{B} 2\left(+34^{\circ}\right)$ & 7.11 & 1.0 & $0.4 \times 0.5$ & -0.0590 & SB1 & 5.599 & 0.5 & 0.24 & 0.25 \\
\hline B3 $\left(-34^{\circ}\right)$ & 11.71 & 1.0 & $0.4 \times 0.5$ & 0.0590 & SB2 & 6.398 & 0.5 & 0.24 & -0.25 \\
\hline Quads & & & (radius) & {$[\mathrm{T} / \mathrm{m}]$} & $\mathrm{SC} 1$ & 8.098 & 0.5 & 0.24 & 0.25 \\
\hline Q1 & 4.51 & 0.3 & 0.4 & -0.18 & $\mathrm{SC} 2$ & 8.897 & 0.5 & 0.24 & -0.25 \\
\hline Q2 & 5.81 & 0.3 & 0.4 & 0.18 & Sector bends & & & $(h \times w)$ & [T] \\
\hline Q3 & 9.11 & 0.3 & 0.4 & -0.25 & $\mathrm{~B} 1\left(-40^{\circ}\right)$ & 2.000 & 1.0 & $0.4 \times 0.5$ & 0.0693 \\
\hline Q4 & 10.41 & 0.3 & 0.4 & 0.30 & $\mathrm{~B} 2\left(+34^{\circ}\right)$ & 4.499 & 1.0 & $0.4 \times 0.5$ & -0.0590 \\
\hline Q5 & 13.71 & 0.3 & 0.4 & -0.26 & $\mathrm{~B} 3\left(-34^{\circ}\right)$ & 6.998 & 1.0 & $0.4 \times 0.5$ & 0.0590 \\
\hline Q6 & 15.01 & 0.3 & 0.4 & 0.28 & Quads & & & (radius) & {$[\mathrm{T} / \mathrm{m}]$} \\
\hline Q7 & 17.23 & 0.3 & 0.4 & 0.20 & Q6 & 9.497 & 0.3 & 0.5 & -0.15 \\
\hline Q8 & 18.04 & 0.3 & 0.4 & -0.46 & Q7 & 11.217 & 0.3 & 0.4 & 0.40 \\
\hline Q9 & 19.58 & 0.3 & 0.4 & 0.40 & Q8 & 12.027 & 0.3 & 0.4 & -0.45 \\
\hline Q10 & 20.88 & 0.3 & 0.4 & -0.40 & Q9 & 13.567 & 0.3 & 0.4 & 0.30 \\
\hline Separator & & & $(h \times w)$ & & Q10 & 14.867 & 0.3 & 0.4 & -0.20 \\
\hline \multirow[t]{2}{*}{$\mathrm{V}$} & 16.32 & 0.7 & $0.2 \times 0.6$ & $350 \mathrm{kV}$ & Separator & & & $(h \times w)$ & \\
\hline & & & & $B_{x}=-0.0215 \mathrm{~T}$ & $\mathrm{~V}$ & 10.307 & 0.7 & $0.2 \times 0.6$ & $360 \mathrm{kV}$ \\
\hline Scrapers & & & (plane) & (setting) & & & & & $B_{x}=-0.0221 \mathrm{~T}$ \\
\hline $\mathrm{C} 1$ & 18.36 & & vert. & $\leq-0.230 \mathrm{~m}$ & Scrapers & & & (plane) & (setting) \\
\hline $\mathrm{C} 2$ & 19.44 & & vert. & $\leq-0.035 \mathrm{~m}$ & $\mathrm{C} 1$ & 12.347 & & vert. & $\leq-0.250 \mathrm{~m}$ \\
\hline & & & & & $\mathrm{C} 2$ & 13.427 & & vert. & $\leq-0.090 \mathrm{~m}$ \\
\hline
\end{tabular}

\section{A. The early version of beam line (Design I) with quad doublets}

The design of the early version of the beam line (reported at IPAC2013 [1] and shown in Fig. 2) is similar to the E4 beam line at PSI [11], but uses quadrupole doublets rather than triplets for focusing. A proton beam hits a thin long carbon target placed midway between solenoids -1 and 1 (see Fig. 7). Surface muons are collected by solenoids 1, 2 and 3 . To reduce the coupling from the three solenoids, we required that

$$
B_{\mathrm{sol}, 1}+B_{\mathrm{sol}, 2}+B_{\mathrm{sol}, 3}=0
$$

The solenoid -1 is used to continue the field lines with $B_{\mathrm{sol},-1}=B_{\mathrm{sol}, 1}$ and may also be used for beginning of a second beam line. While this does not totally eliminate the coupling since the target was located in a nonzero field region, it provides sufficient decoupling downstream at the bends and separator. Parameters of the collection solenoids and other elements are listed in Table II, and apertures are plotted in Fig. 14. 


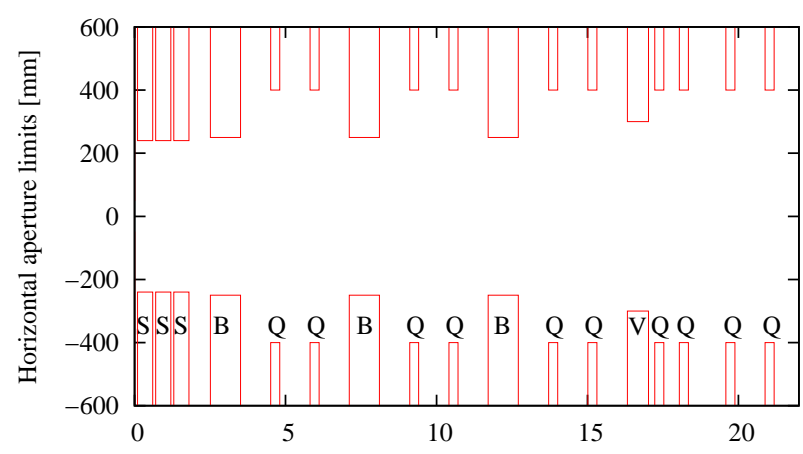

a)

$\mathrm{s}[\mathrm{m}]$

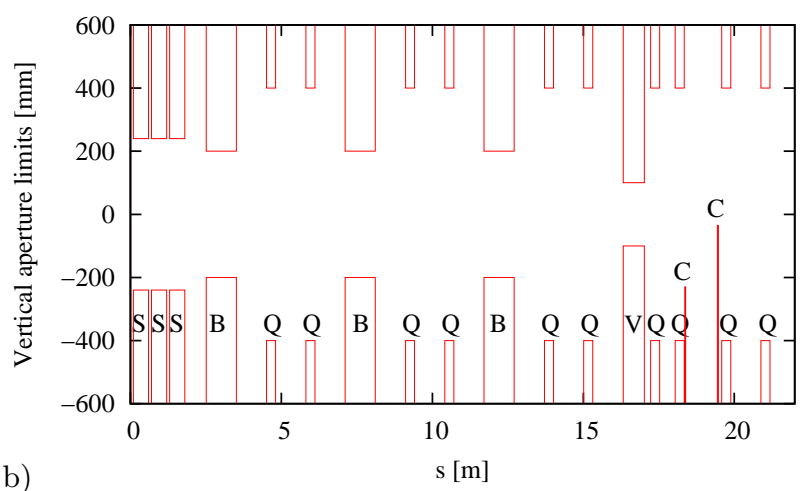

b)

FIG. 14: Aperture limits for the earlier Design I. Element labels are: solenoids "S"; sector bends "B"; quadrupoles "Q"; separator "V"; and scrapers "C". a) Horizontal. b) Vertical. (Solenoids and quadrupoles have circular apertures.)
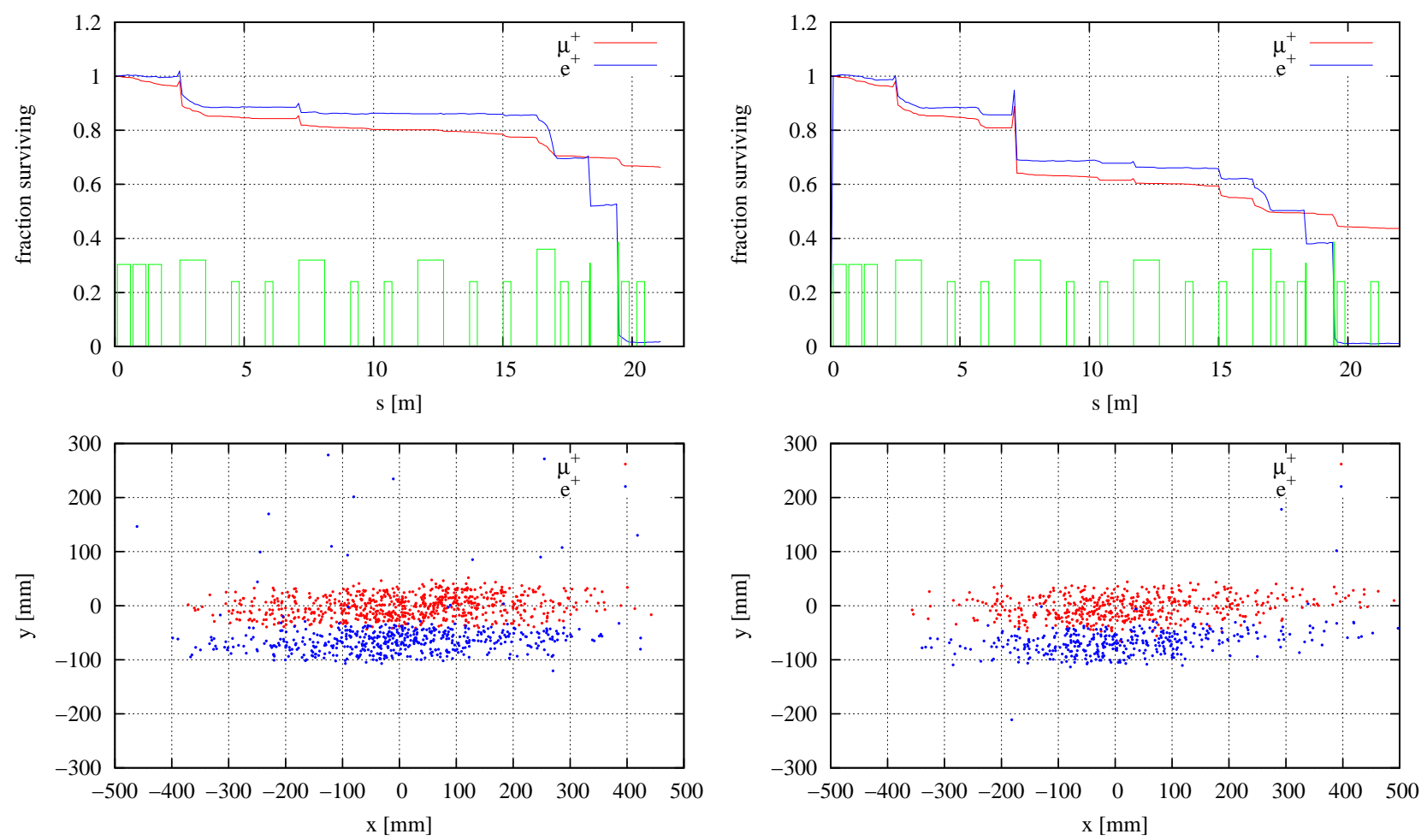

FIG. 15: For Design I, beam loss along the beam line (top plots) and transverse profiles of $\mu^{+}$and $\mathrm{e}^{+}$just upstream of Scraper C1 (bottom). Left: for $\sigma_{p}=0$. Right: for $\sigma_{p}=1.5 \mathrm{MeV} / \mathrm{c}$.

Since the simulation of the production of surface muons from a proton beam hitting a target is very inefficient, requiring days of CPU time for a decent sample, the beam line was optimized with monochromatic particles $\left(\mu^{+}, \mathrm{e}^{+}\right.$, $\pi^{+}, \mathrm{K}^{+}$, and protons) of momentum $27.972 \mathrm{MeV} / \mathrm{c}$ originating just downstream of a $100 \mathrm{~mm}$ long, $50 \mathrm{~mm}$ high, $6 \mathrm{~mm}$ thick graphite target. For this we used a "rectangular" beam generation in G4beamline for a uniform initial position of $100 \times 50 \mathrm{~mm}$ in $x$ and $y$ and with rms divergences: $\sigma_{x^{\prime}}=\sigma_{y^{\prime}}=100 \mathrm{mrad}$. We found that all the $\pi^{+}$(lifetime: $\tau=26 \mathrm{~ns})$ and $\mathrm{K}^{+}(\tau=12 \mathrm{~ns})$ decay well before reaching the separator, and protons are lost inside the scraper (see Fig. 13). Any neutrals or negative particles get removed by the sector bends. What is left are the $\mu^{+}$and some $\mathrm{e}^{+}$ which have been deflected as shown in Fig. 15. 

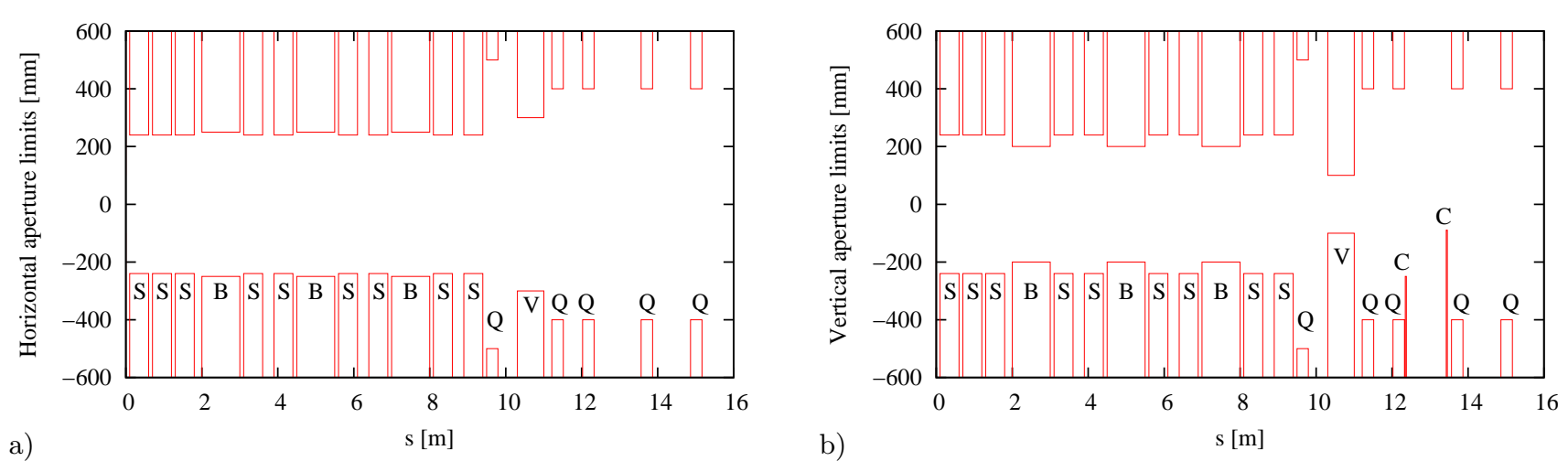

FIG. 16: Aperture limits for the newer Design II. Element labels are: solenoids "S"; sector bends "B"; quadrupoles "Q"; separator "V"; and scrapers "C". a) Horizontal. b) Vertical.
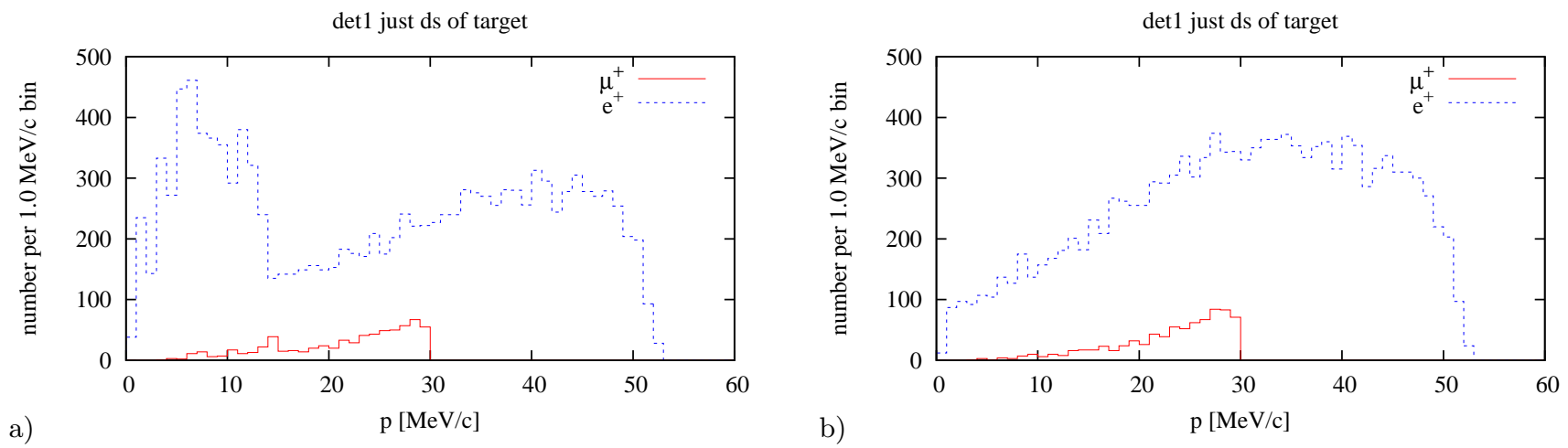

FIG. 17: Momentum distributions of $\mu^{+}$and $\mathrm{e}^{+}$just downstream of $6 \mathrm{~mm}$ thick graphite target collected from $19833 \pi^{+}$at rest evenly spaced in depth and along the $200 \mathrm{~mm}$ length of the target. a) With $B_{\text {sol-1,1,2,3: }} 0.43,0.43,-0.2,-0.23 \mathrm{~T}$ for a total of $679 \mu^{+}$and $12728 \mathrm{e}^{+}$. b) With $B_{\text {sol-1,1,2,3 }}: 1.5,0.59,-0.4,-0.23 \mathrm{~T}$. Many of the low energy e $\mathrm{e}^{+}$below $14 \mathrm{MeV} / \mathrm{c}$ which were seen in a) were removed with the higher fields in b).

\section{B. Most recent version (Design II) of beam line}

More recently, in order to improve the acceptance for the longer $200 \mathrm{~mm}$ target and larger divergences which can be captured with higher solenoid fields of Eqs. 14-17, we have shortened some drifts and replaced some of the quadrupole doublets with pairs of solenoids. The two solenoids of each pair have equal but opposite fields in order to cancel coupling. Parameters for this design are given in Table III and apertures are plotted in Fig. 16 These field values in Table III correspond to a beam of monochromatic muons of momentum $29.792 \mathrm{MeV} / \mathrm{c}$.

\section{OPTIMIZATION OF THE TARGET WIDTH AND THE PROTON BEAM}

As we noted in $\S$ II (see Fig. 5b) surface muons from stopped pions only come from within a depth of $0.7 \mathrm{~mm}$ of a graphite target. Fig. 17 shows the momentum distributions of $\mu^{+}$and $\mathrm{e}^{+}$detected just downstream of a 6 mm thick target for two different settings of the capture solenoids. In both cases the positrons greatly outnumber the muons in each momentum bin. The higher fields discussed in $\S \mathrm{IV}$ remove the broad low momentum $(<14 \mathrm{MeV} / \mathrm{c})$ positron peak in Fig. 17a, and yield an increase for higher momenta for both $\mu^{+}$and $\mathrm{e}^{+}$.

By using a thinner target the positron yield may be decreased relative to the muon yield as demonstrated in Fig. 18 . Reducing the target thickness will also decrease the overall heating of the target. Table IV lists the number of muons and positrons from rest pions that are transported along the beam line of Design II for the two thicknesses. 
det1 just ds of target

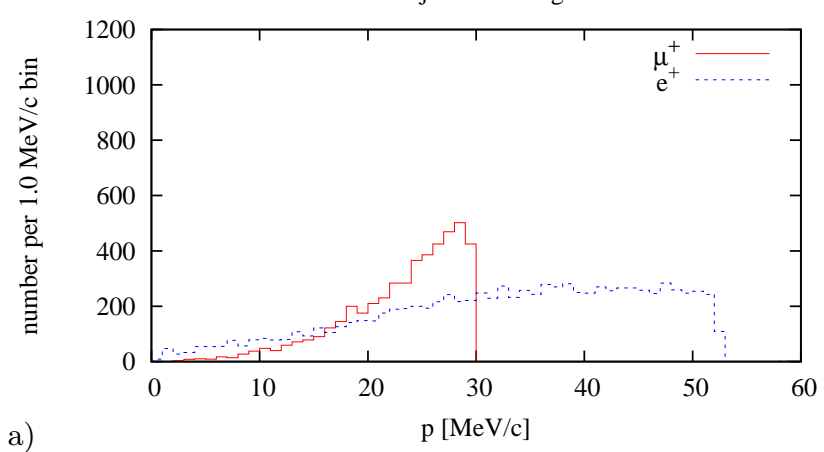

a)

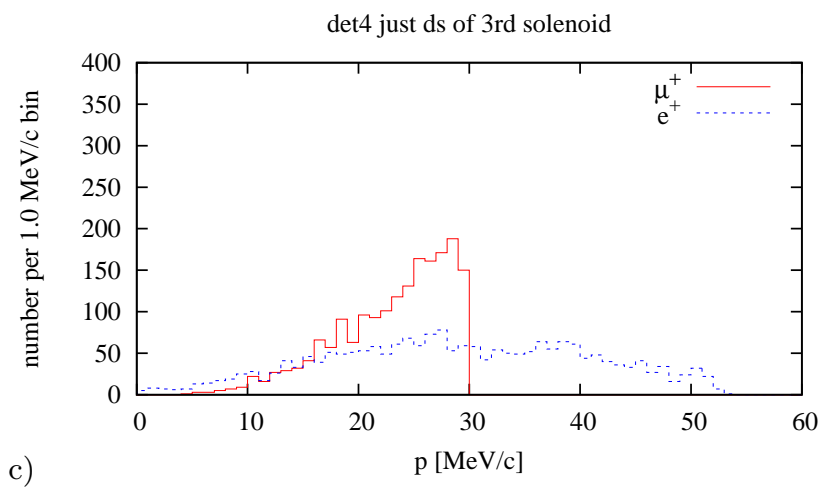

det1 just ds of target
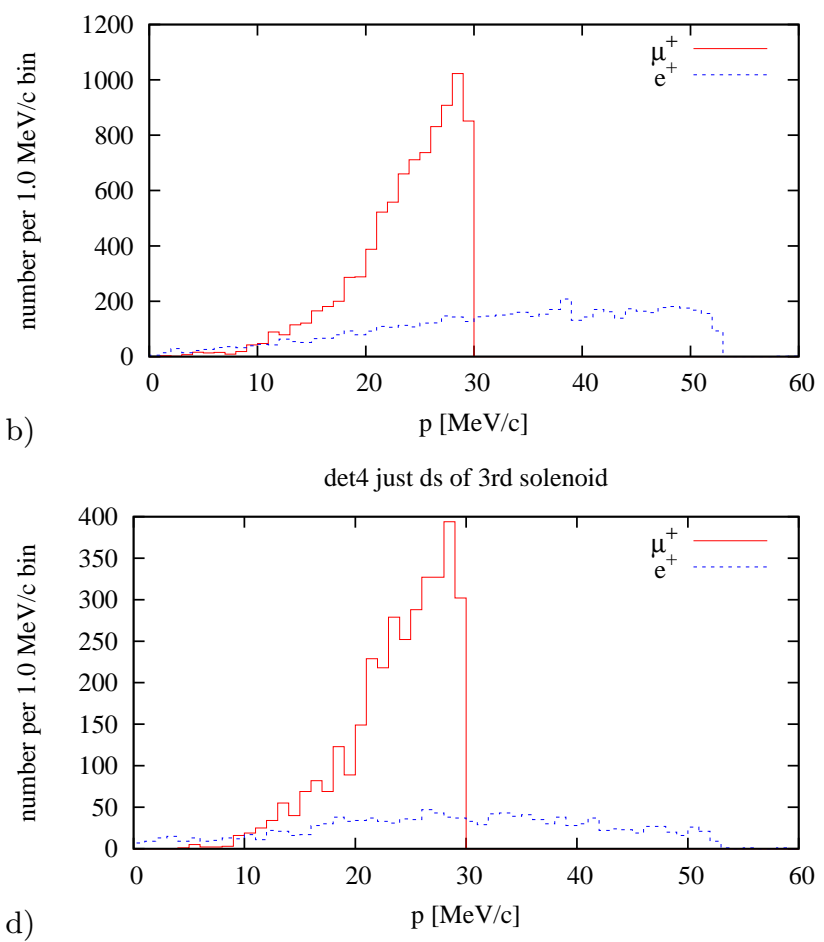

FIG. 18: Momentum distributions of $\mu^{+}$and $\mathrm{e}^{+}$from a uniform distribution of 19836 rest $\pi^{+}$in a graphite target and solenoid fields $B_{\text {sol }-1,1,2,3}: 1.5,0.59,-0.4,-0.23 \mathrm{~T}$. Left: Target width $w_{\text {targ }}=1 \mathrm{~mm}$. Right: Target width $w_{\text {targ }}=0.5 \mathrm{~mm}$. Top: Immediately downstream of target. Bottom: Just after solenoid $\mathrm{S}(3)$.

TABLE IV: Transmission of $\mu^{+}$and $\mathrm{e}^{+}$from 19836 rest pions

\begin{tabular}{crrrrl}
\hline \hline & \multicolumn{3}{c}{$w_{\text {targ }}=1 \mathrm{~mm}$} & \multicolumn{2}{c}{$w_{\text {targ }}=0.5 \mathrm{~mm}$} \\
Det & $\mu^{+}$ & $\mathrm{e}^{+}$ & $\mu^{+}$ & $\mathrm{e}^{+}$ & Where \\
\hline 1 & 4735 & 9337 & 8884 & 5697 & after target \\
4 & 1845 & 2067 & 3399 & 1377 & after 3rd solenoid \\
5 & 670 & 973 & 1271 & 741 & after 1st bend \\
8 & 401 & 344 & 787 & 234 & after 2nd bend \\
11 & 317 & 250 & 618 & 153 & after 3rd bend \\
15 & 153 & 110 & 294 & 76 & after separator \\
20 & 140 & 49 & 260 & 27 & end of beam line \\
\hline
\end{tabular}

\section{A. Proton beam}

With the $0.5 \mathrm{~mm}$ thick, $200 \mathrm{~mm}$ long graphite target, a simulation with a low divergence beam showed a large falloff of secondary particles down the length of the target (see Fig. 19). This beam was produced $401 \mathrm{~mm}$ upstream of the leading target edge by the internal generator of G4beamline with $\sigma_{x}=0.25 \mathrm{~mm}, \sigma_{x^{\prime}}=0.001$, and $\sigma_{y}=10.0 \mathrm{~mm}$, $\sigma_{x^{\prime}}=0.0001$. These emittances are too small for a high intensity proton beam from the AGS $\left(\pi \epsilon_{\mathrm{N}, h}^{95 \%} \sim 3.6 \pi \mu \mathrm{m}\right.$ and $\left.\pi \epsilon_{\mathrm{N}, v}^{95 \%} \sim 14 \pi \mu \mathrm{m}\right)$.

A more realistic beam would have normalized $95 \%$-emittances of $50 \pi \mu \mathrm{m}$ in both planes, and we would also expect to focus the beam onto the target. In order to provide a beam of the correct shape with a waist at the middle of the target, a simple program was written to create a file of beam tracks with the desired emittances and Courant-Snyder parameters. We verified the beam distribution by modeling the target with a small gap in the center and extra detectors along the proton beam as shown in Fig. 20.

The rms beam sizes of the generated beam are shown in Fig. 21a. Horizontal profiles at $x=-100,0$, and $100 \mathrm{~mm}$ in Fig. 21b for a beam of 10,000 protons demonstrate that with no target the waist is centered at $x=0$. A similar computer run with the target used as a perfect absorber (Fig. 21c) shows that a large portion of the protons will pass 
478256 protons on $0.5 \mathrm{~mm}$ thick target; Beam $\sigma_{\mathrm{x}}=0.25 \mathrm{~mm}$

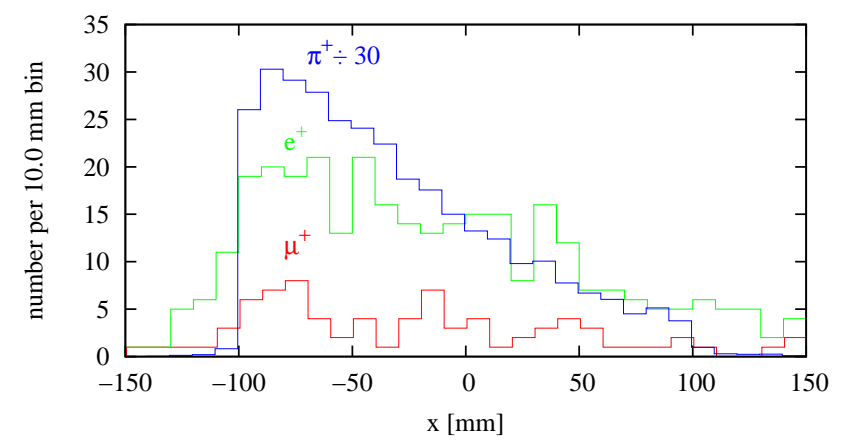

FIG. 19: Distributions of secondary $\pi^{+}, \mathrm{e}^{+}$and $\mu^{+}$from 478256 protons on a graphite target with the low emittance beam.

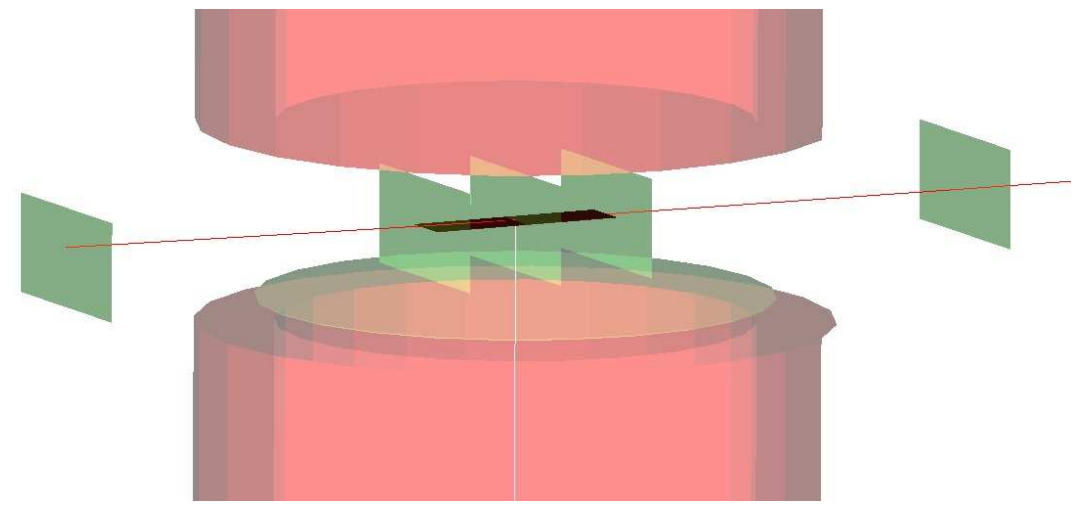

FIG. 20: Layout of five detectors (green) along proton beam hitting thin graphite target $(200 \times 50 \times 0.5 \mathrm{~mm})$. Protons come in from the left along the $x$-axis. The detectors are located at $x=-500,-100,0,100$, and $500 \mathrm{~mm}$.

through the target volume. With the particles allowed to interact in the target, we get the distributions of protons (other secondary particles have been filtered out for this plot) in the detectors as given in Fig. 21d. Comparing the distributions' tails at the ends of the target in b) and d) we see that the multiple horizontal scattering is negligible in the horizontal plane for a beam with this large a divergence.

Using several days of CPU time on an elderly computer, we collected tracks on a virtual detector just downstream of the target for almost $53 \times 10^{6}$ protons on target with solenoid fields as given in Table III. The only track cuts which were instituted for this collection were to remove neutrinos and antineutrinos since their likelihood of interaction is miniscule. We then filtered the generated tracks just downstream of the target for specific particles and obtained a sample of $7536 \mu^{+}$and $31209 \mathrm{e}^{+}$which could be used for tracking through the beam line. Another sample of $1.39 \times 10^{6}$ $\pi^{+}$was also filtered.

Fig. 22a shows the distributions of muons and positrons created along the length of the target. Notice that the centering of the peaks is much better than in Fig. 19. In Fig $22 \mathrm{~b}$ we see that the momentum distribution of muons peaks up above the pions and positrons in the region of interest for surface muons (25 to $30 \mathrm{MeV} / \mathrm{c}$ ).

Since the beam line was initially tuned using monochromatic $\mu^{+}$of momentum $29.792 \mathrm{MeV} / \mathrm{c}$, i. e. the maximum expected from a pion at rest just at the surface of the target, we could expect that the optimum settings for the magnets would be slightly lower for best collection and transport of the muon beam. Fig. 23a shows the momentum distributions at the end of the beam line for a subsample of 36.3 million protons on the target with the beam line set for $29.792 \mathrm{MeV} / \mathrm{c}$. A scan of muon transmission versus field-strength scale factor (see Fig. 23b) which was fit to a parabola gives the optimum field scaling at $94.0 \%$ corresponding to $28.00 \mathrm{MeV} / \mathrm{c}$.

After rescaling the fields, the filtered $\mu^{+}$and $\mathrm{e}^{+}$beams from the full 52.9 million protons were tracked through the beam line with the transmission numbers as listed in Table V. Fig. 24 shows the momentum and $x y$ distributions at the end of the line. The $328 \mu^{+}$and $53 \mathrm{e}^{+}$at the end of the line give a $\mu^{+} / \mathrm{e}^{+}$ratio of 6.2 . For a beam of

$$
15 \times 10^{12} \text { protons } / \text { spill } \times 6.67 \mathrm{~Hz}=10^{14} \text { protons } / \mathrm{s},
$$




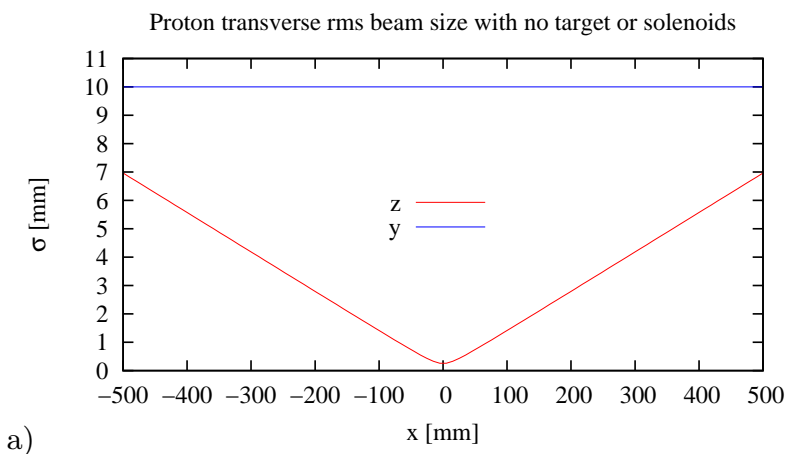

a)

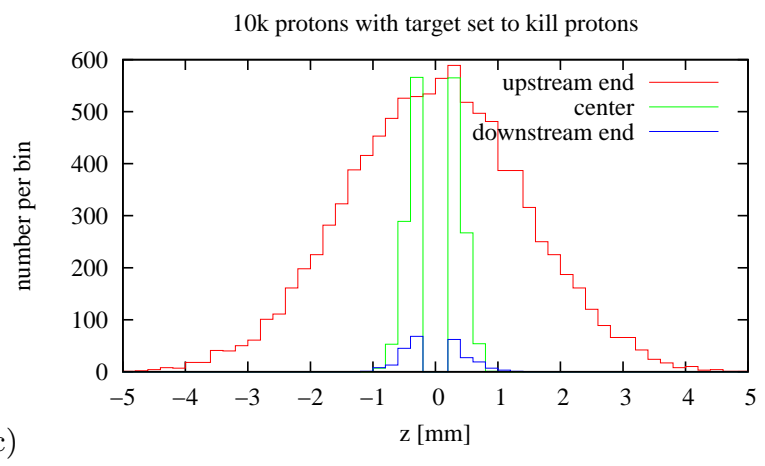

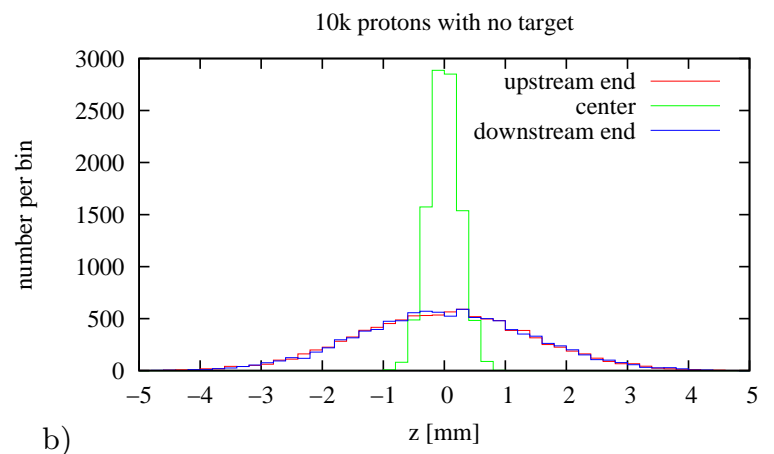

10k protons with $0.5 \mathrm{~mm}$ thick graphite target, no solenoids

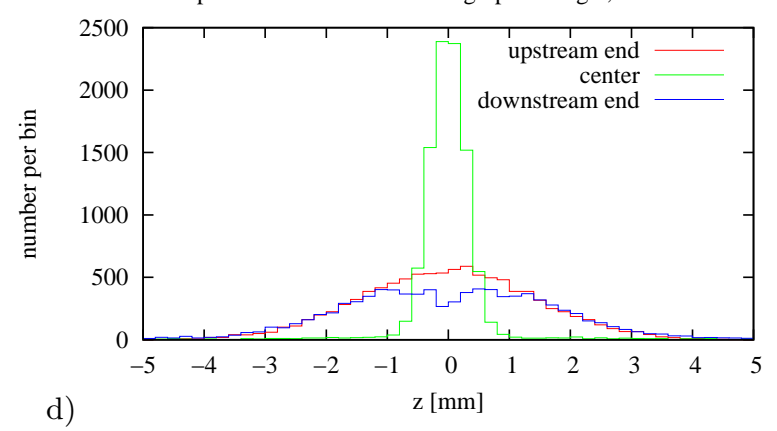

FIG. 21: a) Transverse rms beam size for a $50 \pi \times 10^{-6} \mathrm{~m}$ normalized $95 \%$ emittance proton beam used in the simulations with a $0.5 \mathrm{~mm}$ thick $200 \mathrm{~mm}$ long graphite target. b) Horizontal beam profile at $x=-100$, 0 , and $100 \mathrm{~mm}$ without the target. c) Similar horizontal profiles, but with protons removed when they intercept the target volume. d) Profiles with the protons allowed to interact with the target; only protons are shown in the distributions. Note that the horizontal divergence is large enough that multiple scattering is negligible as can be seen from a comparison of the tails in plots $b$ and $d$.
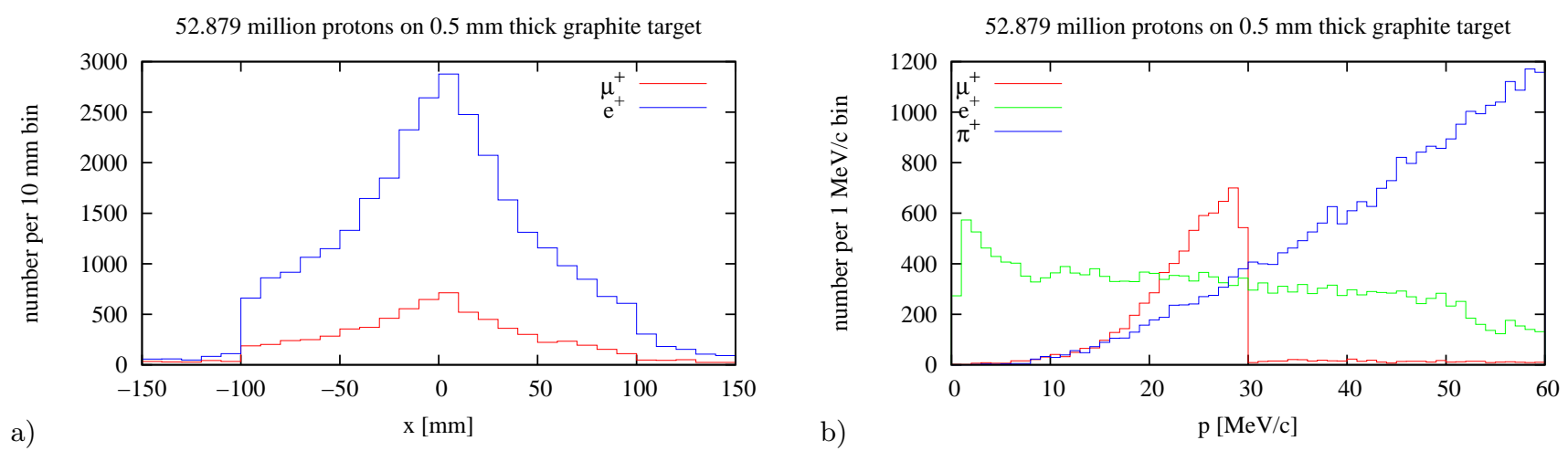

FIG. 22: Distributions of filtered tracks from almost 52.9 million protons on target as detected $0.2 \mathrm{~mm}$ from the surface of the target. a) Number of $\mu^{+}$and $\mathrm{e}^{+}$produced along the length of the target. b) Momentum distributions of $\mu^{+}$, $\mathrm{e}^{+}$, and $\pi^{+}$below $60 \mathrm{MeV} / \mathrm{c}$.

TABLE V: Transmission of $\mu^{+}$and $\mathrm{e}^{+}$

\begin{tabular}{rrrl}
\hline \hline Det & $\mu^{+}$ & $\mathrm{e}^{+}$ & Where \\
\hline 1 & 7512 & 32526 & after target \\
4 & 2606 & 4542 & after 3rd solenoid \\
5 & 1358 & 1338 & after 1st bend \\
8 & 953 & 460 & after 2nd bend \\
11 & 781 & 311 & after 3rd bend \\
15 & 385 & 158 & after separator \\
20 & 328 & 53 & end of beam line \\
\hline
\end{tabular}


$\operatorname{det} 20$ at end of line: $36.3 \mathrm{M}$ protons on $0.5 \mathrm{~mm}$ graphite target

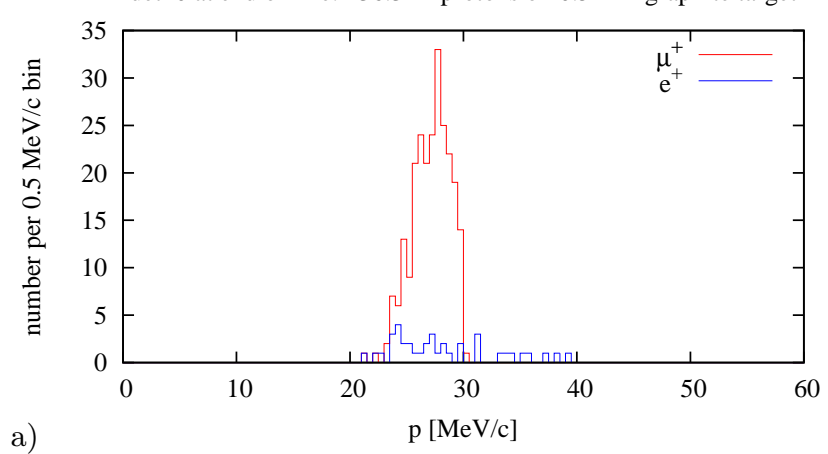

Field scan for 36.3 million protons

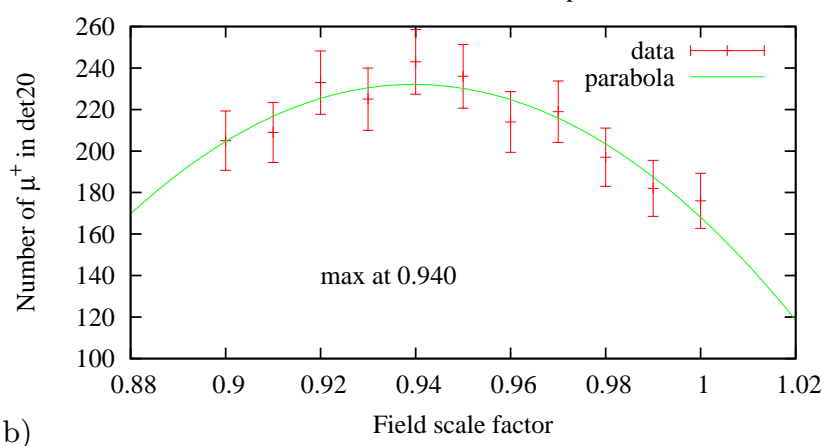

FIG. 23: a) Momentum distributions for muons and positrons at the end of the beam line for a subsample of 36.3 million proton events. The EM fields were set as listed in Table III for a momentum $29.792 \mathrm{MeV} / \mathrm{c}$. b) A scan of transmission versus overall field scaling showing that for optimum transmission the fields should be scaled down by a factor of 0.94 .
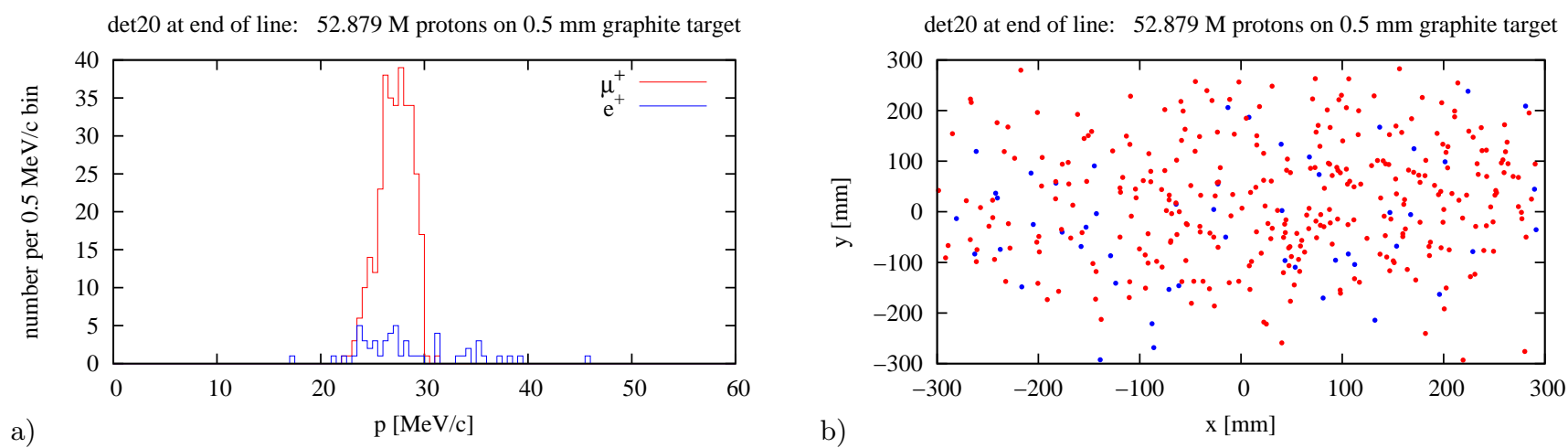

FIG. 24: Results at the end of the beam line after rescaling fields by 0.94 . a) Momentum distributions for muons and positrons at the end of the beam line for about 52.9 million protons on target. b) The corresponding $x y$-distribution of $\mu^{+}$(red) and $\mathrm{e}^{+}$ (blue) tracks at the end of the beam line.

these scale yields of

$$
328 \times \frac{100 \times 10^{12}}{53 \times 10^{6}}=620 \mathrm{MHz} \text { for muons, }
$$

and $100 \mathrm{MHz}$ for positrons at then end of the beam line.

By using a thin $0.5 \mathrm{~mm}$ thick graphite target, we greatly reduce the production of positrons while keeping the surface muon production high. This thinner target also reduces the heating of the target, so that it should easily be radiatively cooled. This does require a proton beam which is focused onto the center of the target with a high horizontal divergence; the high divergence also minimizes the effect of multiple scattering of the proton beam.

\section{B. Target heating}

A simulation with 10,000 protons on target gave the amount of energy deposited in the target and solenoids as shown in Table VI. The power deposited for a rate of $10^{14}$ proton per second is also shown with the target receiving about $500 \mathrm{~W}$ of heating from the beam.

To understand the target heating profile, we divided the target into $9.9 \mathrm{~mm}$ strips separated by $0.1 \mathrm{~mm}$ gaps and ran a simulation with $10^{5}$ protons and no magnets. The result is the histogram of deposited power scaled for $10^{14}$ protons per second shown in Fig. 25. The summed power from all twenty strips was $498 \mathrm{~W}$ which would should be scaled up to $503 \mathrm{~W}$ to account for the $1 \%$ of graphite which should fill in the gaps.

Ignoring conduction and only considering radiation, we may estimate the maximum temperature $T$ from the Stefan- 


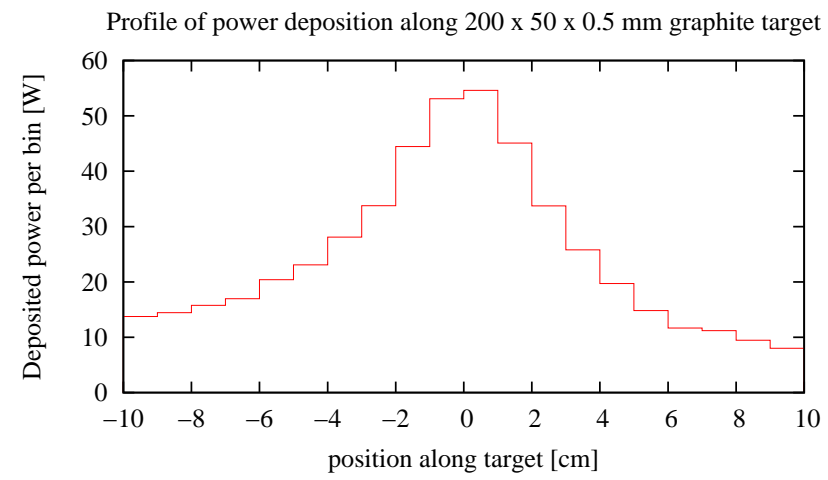

FIG. 25: Profile of power deposition along the target assuming a $1.5 \mathrm{GeV}$ kinetic energy proton beam of $10^{14}$ protons/s with a normalized $95 \%$ emittance of $50 \pi \times 10^{-6} \mathrm{~m}$. This assumes horizontal and vertical waists of $\sigma_{\mathrm{h}}=0.25 \mathrm{~mm}$ and $\sigma_{\mathrm{v}}=10 \mathrm{~mm}$ in the center of the target. Not surprisingly, the shape of this profile is very similar to the $x$-profiles of Fig. 22.

TABLE VI: Deposition of Power in target and solenoids

\begin{tabular}{lccc}
\hline Element & $\begin{array}{c}\text { Deposited energy } \\
\text { Total }^{b} \\
{[\mathrm{GeV}]}\end{array}$ & $\begin{array}{c}\text { Power }^{a} \\
\text { peroton } \\
{[\mathrm{MeV}]}\end{array}$ & $\begin{array}{c}\left(10^{14} \mathrm{p} / \mathrm{s}\right) \\
{[\mathrm{W}]}\end{array}$ \\
\hline Target & 318 & 31.8 & 508 \\
Sol(-1) & 118 & 11.8 & 189 \\
Sol(1) & 112 & 11.2 & 180 \\
Sol(2) & 5.7 & 0.57 & 9.1 \\
Sol(3) & 1.1 & 0.11 & 1.7 \\
Sol(A1) & 0.03 & 0.03 & 0.5 \\
\hline
\end{tabular}

${ }^{a}$ Scaled to $10^{14}$ protons/s.

${ }^{b}$ Simulated for a total of $10^{4}$ protons.

Boltzmann law for black-body radiated power

$$
P=\epsilon \sigma A T^{4}
$$

where $\sigma=1.38 \times 10^{-23} \mathrm{~J} / \mathrm{K}$, assuming an emissivity $\epsilon=0.98$ for graphite and area

$$
A=\left(\frac{200 \mathrm{~mm}}{20}-0.1 \mathrm{~mm}\right) \times 50 \mathrm{~mm} \times 2=0.00099 \mathrm{~m}^{-2} .
$$

With $P=55 \mathrm{~W}$ in the central $1 \mathrm{~cm}$ strip, we obtain $T_{\max } \simeq 960 \mathrm{~K}$ for a rate of $10^{14}$ protons per second. For a reduced emissivity[4] of 0.8 , this temperature would only increase to $1010 \mathrm{~K}$. Any sublimation from graphite at this temperature is probably insignificant since graphite with a temperature of $1600 \mathrm{~K}$ has only a vapor pressure of around $10^{-9}$ Torr [14] and drops off quite substantially for lower temperatures[4, 14].

\section{REDUCING THE HEIGHT OF THE PROTON BEAM}

By reducing the height of the proton beam on the target we may increase the number of muons passing through the aperture of the separator and reaching the last detector. To do this, we first calculated a reference trajectory for the proton beam passing through the magnetic field of the capture solenoids. The reference trajectory was fit to a parabola $y_{r}(x)=a+b x+c x^{2}$ which was then subtracted from our previous samples of $7536 \mu^{+}$and $31209 \mathrm{e}^{+}$ (see Fig. 26) that had been collected from $52.9 \times 10^{6}$ protons with a vertical waist of $\sigma_{y}^{*}(x=0)=10 \mathrm{~mm}$. These differences $\Delta y_{i}=y_{i}-y_{r}\left(x_{i}\right)$, for the $i^{\text {th }}$ particle, were then scaled to a distribution with $\sigma_{y}^{*}=1 \mathrm{~mm}$ by multiplying by square root of the ratio of beta-functions (for a $1 \mathrm{~mm}$ waist instead of the original $10 \mathrm{~mm}$ waist). Adding back the reference trajectory $y_{r}(x)$, we obtained the green distribution shown in Fig. 26a. Finally, to simulate the proton beam being steered onto the target's center, we added a straight line $y(x)=A+B x$ to the distribution (Fig. 26b).

With an additional $100 \mathrm{~cm}^{2}$ area circular detector (radius $=56.4 \mathrm{~mm}$ ) placed $10 \mathrm{~mm}$ behind the $20^{\text {th }}$ detector (at $s=16.176 \mathrm{~m}$ ), we retuned the last pair of quadrupoles (see last column of Table VII) to peak up the muon intensity 

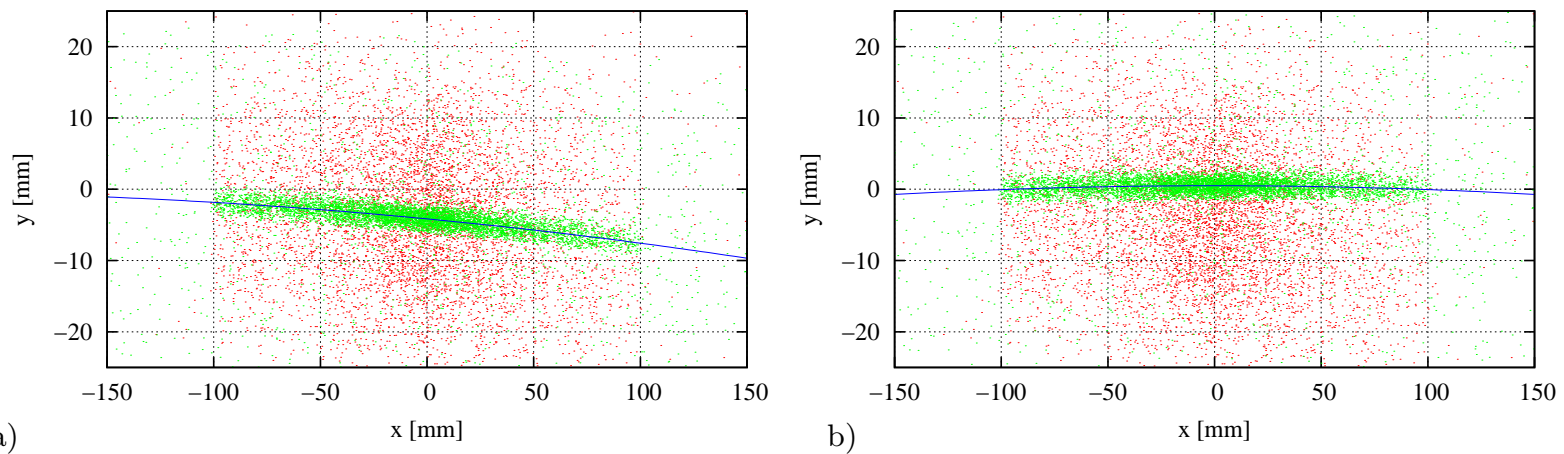

FIG. 26: a) The blue curve is reference trajectory of the proton beam. Red points are the distribution of $7536 \mu^{+}$just downstream of the target (at $z=0.45 \mathrm{~mm}$ ) for a proton beam with $\sigma_{y}^{*}=10 \mathrm{~mm}$. Green points are scaled to correspond to $\sigma_{y}^{*}=1 \mathrm{~mm}$ as described in the text. b) Proton reference and scaled (green) points shifted linearly to the center line of the target $(y=0)$.
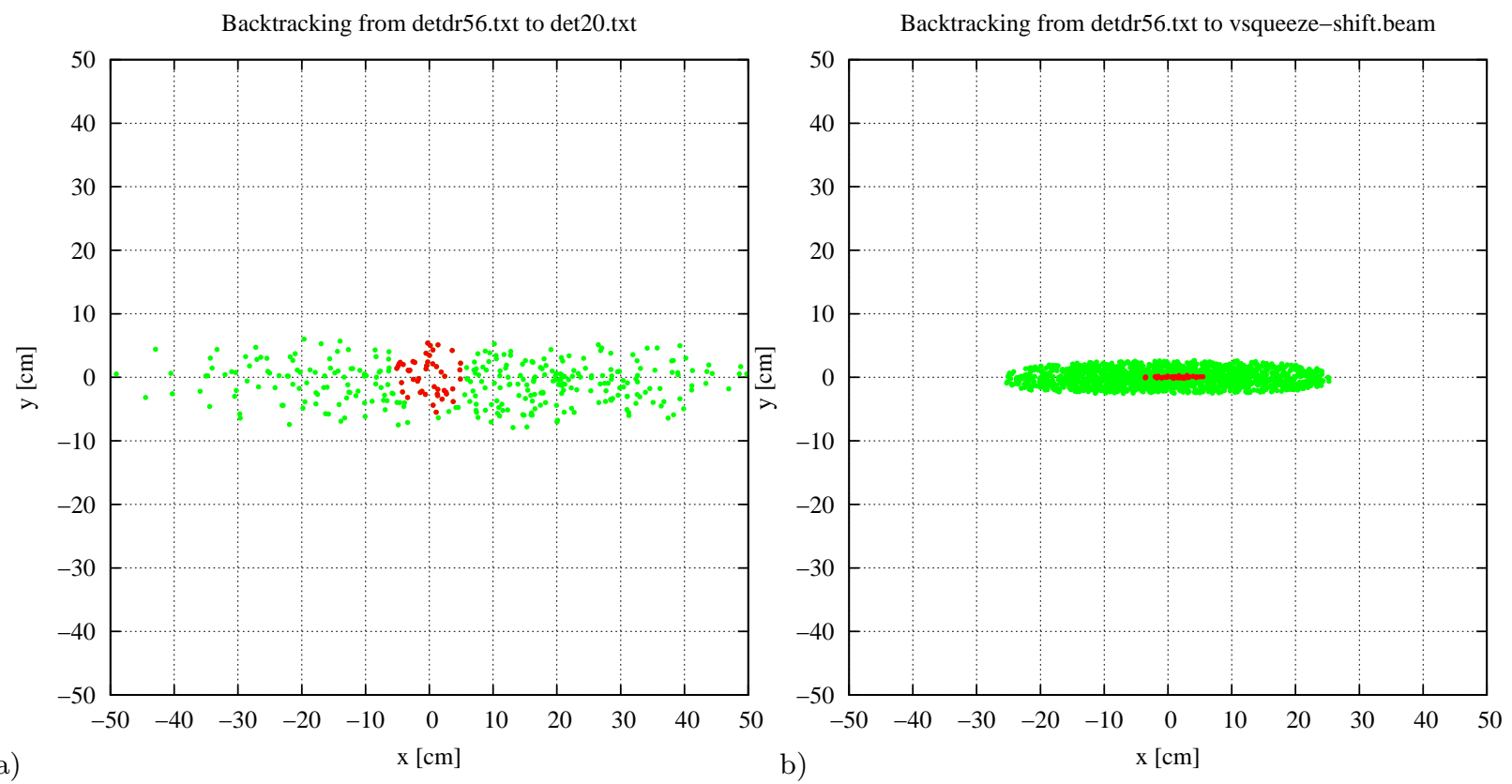

FIG. 27: a) Red (green) points are $\mu^{+}$in detector 20 which hit (missed) the smaller detector 21. b) Red (green) points are $\mu^{+}$ from the upstream beam sample of $7536 \mu^{+} 0.2 \mathrm{~mm}$ from the surface of the target. which hit (missed) the smaller

at the end of the beam line. This gave 349 and $48 \mu^{+}$respectively in detectors 20 and 21 (see Fig. 27). The respective numbers of positrons were 48 and 7 for the two detectors. Table VIII lists the total rate of muons and positrons expected at the end of the line in detector 20, as well as the flux per square centimeter from the smaller detector 21. Fig. 27b shows that backtracking the final muons in detector 21 back to the target indicates that a shorter target (10-15 cm long) could be used.

TABLE VII: Quad strengths for $p_{\text {ref }}=28 \mathrm{MeV} / \mathrm{c}$

\begin{tabular}{lrr}
\hline \hline Quad & $\begin{array}{c}\text { Table III } \\
{[\mathrm{T} / \mathrm{m}]}\end{array}$ & $\begin{array}{r}\text { retuned } \\
{[\mathrm{T} / \mathrm{m}]}\end{array}$ \\
\hline Q6 & -0.141 & -0.141 \\
Q7 & 0.376 & 0.376 \\
Q8 & -0.423 & -0.423 \\
Q9 & 0.282 & 0.380 \\
Q10 & -0.188 & -0.450 \\
\hline
\end{tabular}

TABLE VIII: Muon and positron rates

\begin{tabular}{lcc}
\hline \hline & $\begin{array}{c}\text { det20 } \\
{[\mathrm{MHz}]}\end{array}$ & $\begin{array}{c}\text { det21 } \\
{\left[\mathrm{MHz} / \mathrm{cm}^{2}\right]}\end{array}$ \\
\hline$\mu^{+}$ & 660 & 0.91 \\
$\mathrm{e}^{+}$ & 91 & 0.13 \\
\hline
\end{tabular}




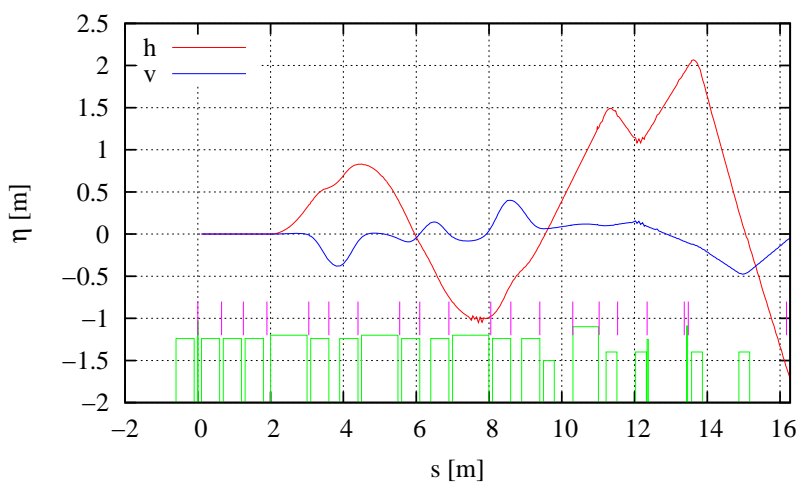

FIG. 28: Horizontal and vertical dispersion functions. The magenta tic marks indicate the longitudinal locations of the 20 detectors.

\section{CONCLUSIONS AND COMMENTS}

We have designed a beam line for the production of surface muons which could be used at BNL using the proton linac, Booster, and AGS as a $1.5 \mathrm{GeV}$ (kinetic energy) proton source. Two variations were presented, with the second improved design having a length of $15.2 \mathrm{~m}$ and longer $200 \mathrm{~mm}$ target. Assuming a proton rate of $10^{14}$ protons per second, we estimate the rate of $\mu^{+}$at the end of the line to be $660 \mathrm{MHz}$, with a contamination of positrons at $91 \mathrm{MHz}$, thus giving a muon to positron ratio of 7 . This $\mu^{+}$rate is higher than the demonstrated rates for the four existing sources (at RAL, J-PARC, TRIUMF, and PSI); however the beam is transversely quite large at the end of the line and is not completely usable for a typical experiment.

With a $2 \mathrm{~mA}$ proton beam $\left(1.25 \times 10^{16}\right.$ protons $\left./ \mathrm{s}\right)$ in the $\mu \mathrm{E} 4$ beam line at PSI[11], we estimate their flux to be about $22 \times 10^{6} \mathrm{~cm}^{-2} \mathrm{~s}^{-1}$ (averaged over $9 \mathrm{~cm}^{2}$ ), or $1.7 \times 10^{-9} \mu^{+} \mathrm{cm}^{-2} \mathrm{~s}^{-1} /$ proton. For our Design II beam line, the corresponding flux per proton is $8.7 \times 10^{-9} \mu^{+} \mathrm{cm}^{-2} \mathrm{~s}^{-1}$ - five times larger.

By using a $0.5 \mathrm{~mm}$ thick graphite target, we can minimize the production of background particles while keeping the $\mu^{+}$production high, thus greatly reducing the number of positrons produced. Additionally the energy deposited in a thinner target $(500 \mathrm{~W})$ can be cooled through radiation. The thin target will require a high divergence proton beam which should be focused with a $\sigma_{h}=0.25 \mathrm{~mm}$ waist at middle of the target.

With the final field strengths, the separator does not seem to work as well as hoped. This is partly due to a shift of the vertical dispersion peak after massive retuning of the line; the first scraper (C1) was originally located at the peak of the vertical dispersion at quadrupole Q8, but now the peak is at the last Q10 as shown in Fig. 28. Clearly some more optimization of the beam line should increase the $\mu^{+} / \mathrm{e}^{+}$ratio at the end of the line. Changing the bend angles (particularly reducing B3) could reduce the horizontal dispersion and aperture requirement at the separator.

\section{APPENDIX: SYRK4TRACK PROGRAM}

The $\mathrm{C}++$ program syrk4track was originally written to track the trajectory and spin through the helical dipoles of the RHIC Siberian snakes and spin rotators. Unlike magnets with transverse magnetic fields which can be specified by a vector potential with only a longitudinal component, the vector potential for a helical dipole has both transverse and longitudinal components. The Hamiltonian

$$
H\left(x, w_{x}, y, w_{y}, z, w_{z} ; c t\right)=\sqrt{\left(\vec{w}-\frac{q \vec{A}}{p_{0}}\right)^{2}+\left(\frac{m c}{p_{0}}\right)^{2}}
$$

where the canonical momentum is given by

$$
\vec{w}=\frac{\vec{P}}{p_{0}}=\frac{\vec{p}+q \vec{A}}{p_{0}}
$$


leads to the equations of motion:

$$
\begin{aligned}
& \frac{1}{c} \frac{d \vec{x}}{d t}=\frac{c}{H}\left(\vec{w}-\frac{q \vec{A}}{p_{0}}\right)=\vec{\beta}, \\
& \frac{1}{c} \frac{d \vec{w}}{d t}=\frac{q}{p_{0}} \sum_{j=1}^{3} \beta_{j} \nabla A_{j} .
\end{aligned}
$$

For a reference trajectory the integration is done by a 4th-order implicit Runge-Kutta integrator using a GaussLegendre algorithm. However for a trajectory along the axis of a set of coaxial solenoids, the choice of integrator for the reference trajectory is really unimportant since the magnetic field is parallel to the trajectory along the axis.

To obtain a transport matrix along the reference trajectory a matrix of second partial derivatives of the Hamiltonian

$$
C_{i j}(t)=\frac{\partial^{2} H}{\partial X_{i} \partial X_{j}}
$$

Then for a small integration step $c \delta t$, the symplectic transport matrix is calculated from the recursion

$$
\mathbf{M}\left(t_{n+1}\right)=e^{\mathbf{S C}(t) c \delta t} \mathbf{M}\left(t_{n}\right)
$$

starting from the identity matrix $\mathbf{M}\left(t_{0}\right)=\mathbf{I}$.

\section{APPENDIX: SOLENOID BEND}

One other beam line design which was briefly considered replaced the sector bends of Design II with a solenoidal bend composed of short solenoids segments with each successive segment rotated about the vertical. Fig. 29 shows an example of a $40^{\circ}$ bend made from eight solenoid segments. Alternating the sign of the field in the segments gave considerable loss of particles. With the fields aligned in the same direction, there is a net coupling in each bend. The vertical drift seen in Fig. 29b can be compensated by tilting each segment slightly about the radial axis; however this tilt depends on the field strength.

Fig. 30 shows a simulation of $\mu^{+}$(dark blue) and $\mathrm{e}^{+}$(cyan) passing through a section of beam line with three solenoid bends. Here the longer solenoid doublets between the bends are used for focusing the beam in the straight sections and each pair has opposing fields. We did not pursue this type of bend, since it seems that tuning the field strengths could be problematic with the beam wandering around considerably. There is no solid reference trajectory for the solenoid bend, such as we have for a more conventional dipole bend.

\section{ACKNOWLEDGMENTS}

We would like to thank the other authors of the IPAC2013 paper[1], J. Alessi, K. Brown, C. J. Gardner, H. Huang, D. Raparia, and T. Roser, for their useful input on the injector system. 

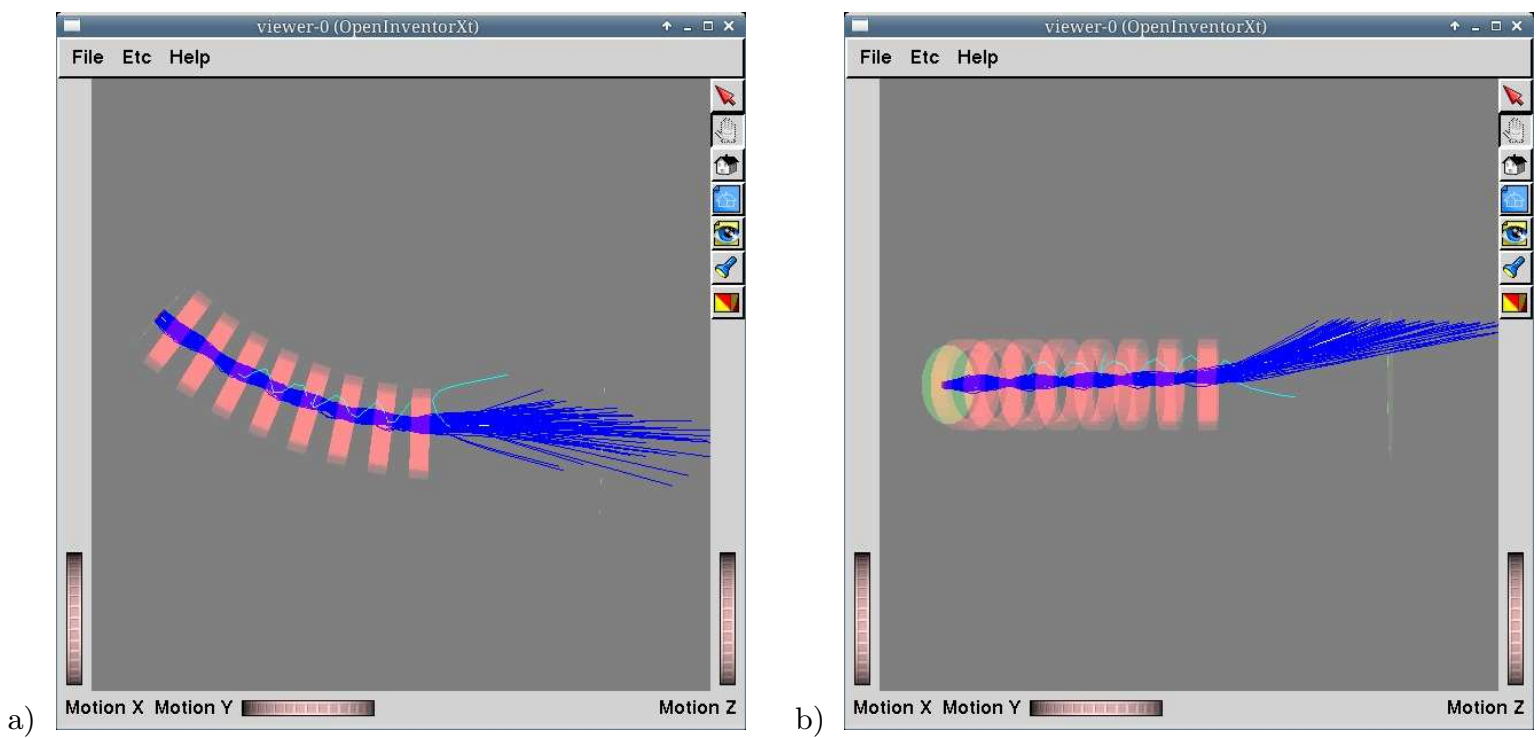

FIG. 29: A $40^{\circ}$ bend composed of eight short solenoid segments of length $12.5 \mathrm{~cm}$ and inner radius of $24 \mathrm{~cm}$. Trajectories are for monochromatic $\mu^{+}$of $29.792 \mathrm{MeV} / \mathrm{c}$ and a central solenoid field of $1.5 \mathrm{~T}$. a) Top down view. b) Side view. Notice that there is a vertical drift of the beam along the bend.

[1] Blaskiewicz, M. et al. (2013). Proposal for a $\mu$ SR Facility at BNL, in Proceedings of IPAC2013, p. 3749.

[2] Conte, M. and MacKay, W. W. (2008). An Introduction to the Physics of Particle Accelerators, 2nd edn. (World Sci., Singapore).

[3] De Renzi, R. (2007). "Introduction to $\mu$ SR", in P. Carretta (ed.), NMR-MRI, $\mu$ SR and Mossbauer Spectroscopies in Molecular Magnets (Springer, Milan), p. 149.

[4] Haines, J. R. and Tsai, C. C. (2002). Graphite sublimation tests for the Muon Collider/Neutrino Factory target development program, Tech. Rep. ORNL/TM-2002/27, ORNL.

[5] Kossler, W. J. (1981). " $\mu$ SR at the AGS at BNL", Hyperfine Interactions 8, p. 797.

[6] Larsen, R. (1971). Transport matrices for a magnetic solenoid, Tech. Rep. SPEAR-107, SLAC.

[7] MacKay, W. W. and Conte, M. (2012). Accelerator Physics Example Problems with Solutions (World Sci., Singapore).

[8] Miyadera, H. et al. (2007). Design of muon accelerators for an advanced muon facility, in Proceedings of PAC0\%, p. 3032.

[9] Miyake, Y. (2013). "J-PARC MLF MUSE (MUon Science Establishment)", BNL Seminar on 29 May, 2013.

[10] Miyake, Y. et al. (2013). Ultra slow muon microspcopy by laser resonant ionization at J-PARC, MUSE, Hyperfine Interactions 216, p. 79 .

[11] Prokscha, T. et al. (2008). The new $\mu \mathrm{E} 4$ beam at PSI: A hybrid-type large acceptance channel for the generation of a high intensity surface-muon beam, Nucl. Inst. and Meth. A595, p. 317.

[12] Roberts, T. (2012). G4beamline users guide 2.12, Tech. rep., Muons Inc.

[13] Shiroka, T. (1999). Spin-Polarized Epithermal Muons: Construction and Test of a Pulsed Source with Applications to Films and Nanostructures, Ph.D. thesis, Universita Delgi Studi Di Parma.

[14] Thieberger, P. (2000). Upper limits for sublimation losses from hot carbon targets in vacuum and gasses, Tech. Rep. MUC-0186, BNL.

[15] Wien, K. (1999). 100 years of ion beams: Willy Wien's canal rays, Brazillian Journal of Physics 29, p. 401.

[16] Wien, W. (1898). Untersuchungen über die electrische Entladung in verdünnten Gasen, Annalen der Physik 301, p. 440. 

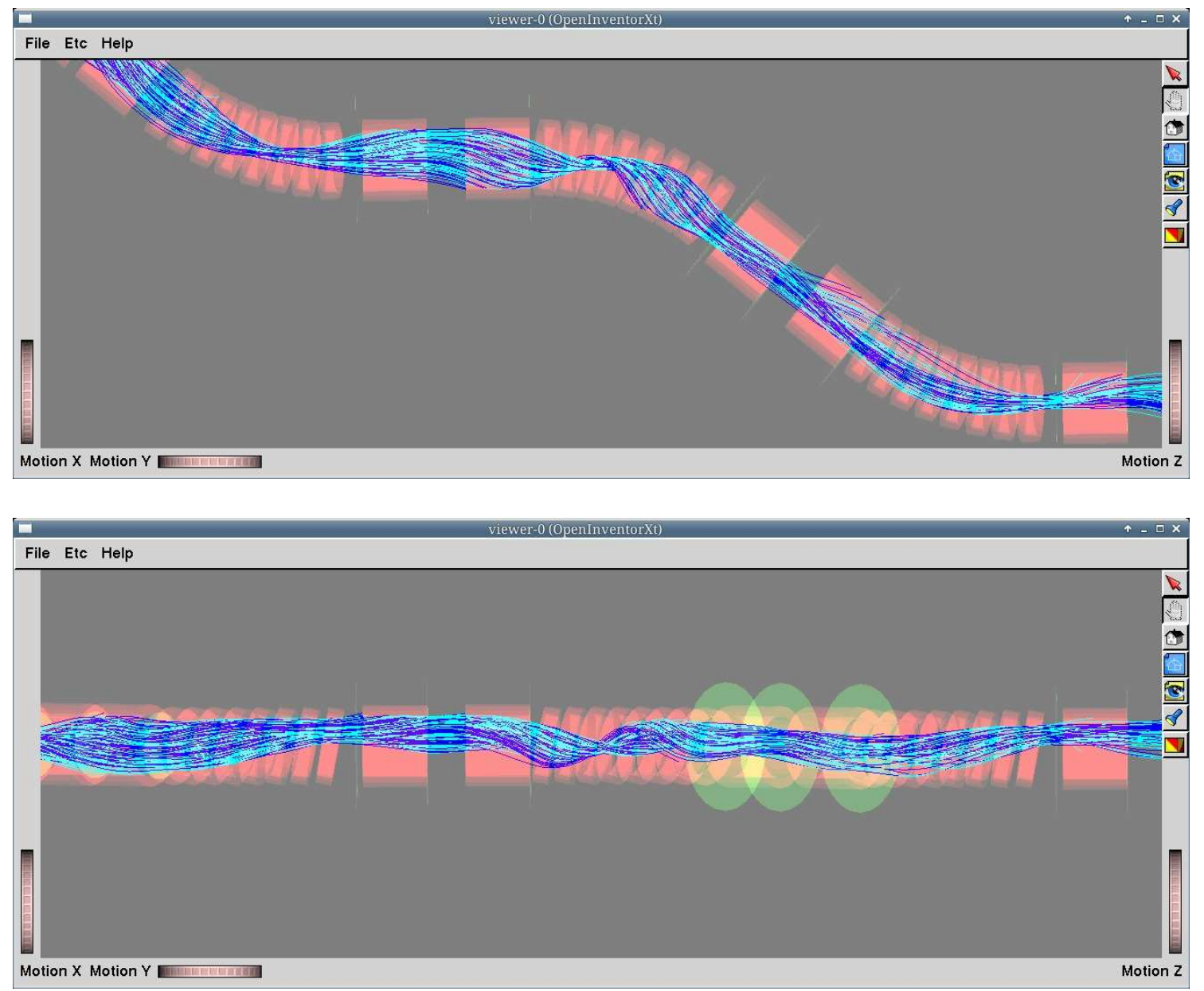

FIG. 30: Section of beam line with three $40^{\circ} 4$ bends separated by solenoid doublets. Here the field in the bends is $0.6 \mathrm{~T}$ with a $8^{\circ}$ radial rotation of solenoid segment Top: Horizontal. Bottom: Vertical. 